\title{
Bidirectional Plasticity of Intrinsic Excitability Controls Sensory Inputs Efficiency in Layer 5 Barrel Cortex Neurons in Vivo
}

\author{
Séverine Mahon ${ }^{1}$ and Stéphane Charpier ${ }^{1,2}$ \\ ${ }^{1}$ Centre de Recherche de l'Institut du Cerveau et de la Moelle épinière, Université Pierre et Marie Curie (UPMC), INSERM UMR-S 975, CNRS UMR 7225, \\ Hôpital Pitié-Salpêtrière, F-75013, Paris, France, and 2UPMC Université Paris 06, F-75005, Paris, France
}

\begin{abstract}
Responsiveness of cortical neurons to sensory inputs can be altered by experience and learning. While synaptic plasticity is generally proposed as the underlying cellular mechanism, possible contributions of activity-dependent changes in intrinsic excitability remain poorly investigated. Here, we show that periods of rhythmic firing in rat barrel cortex layer 5 pyramidal neurons can trigger a long-lasting increase or decrease in their membrane excitability in vivo. Potentiation of cortical excitability consisted of an increased firing in response to intracellular stimulation and a reduction in threshold current for spike initiation. Conversely, depression of cortical excitability was evidenced by an augmented firing threshold leading to a reduced current-evoked spiking. The direction of plasticity depended on the baseline level of spontaneous firing rate and cell excitability. We also found that changes in intrinsic excitability were accompanied by corresponding modifications in the effectiveness of sensory inputs. Potentiation and depression of cortical neuron excitability resulted, respectively, in an increased or decreased firing probability on whisker-evoked synaptic responses, without modifications in the synaptic strength itself. These data suggest that bidirectional intrinsic plasticity could play an important role in experience-dependent refinement of sensory cortical networks.
\end{abstract}

\section{Introduction}

Information processing in neocortical neurons is modulated by sensory experience and learning. Integrative properties of barrel cortex neurons remain highly plastic throughout life, making the rodent whisker system an attractive model for investigating the cellular mechanisms of experience-dependent plasticity (Petersen, 2007; Feldman, 2009). In the adult somatosensory cortex, the preferential use of a subset of whiskers (Diamond et al., 1993; 1994), or the pairing of whisker stimuli with positive or negative reinforcement (Siucinska and Kossut, 1996; 2004), alter the neuronal responses to the trained whiskers. Changes in responsive properties of layer 5 cortical neurons also occur during learning of a whisker-dependent discrimination task and contribute to improvement in the discrimination performance (Wiest et al., 2010). This adaptation of cortical responsiveness likely involves plasticity of synaptic connections within the different layers of barrel cortex (Fox, 2002; Jacob et al., 2007, Skibinska-Kijek et al., 2008; Feldman, 2009).

Received Jan. 27, 2012; revised June 18, 2012; accepted June 25, 2012.

Author contributions: S.M. and S.C. designed research; S.M. performed research; S.M. analyzed data; S.M. and S.C. wrote the paper.

This work was supported by grants from the Fondation pour la Recherche sur le Cerveau, the Institut National de la Santé Et de la Recherche Médicale, and the Pierre and Marie Curie University. We thank R. Miles, P. Faure, G. Huberfeld, M. Pidoux, M. Chipaux, and T. Altwegg-Boussac for constructive discussions and helpful comments on this manuscript.

Correspondence should be addressed to Séverine Mahon, Institut du Cerveau et de la Moelle épinière, Hôpital Pitié-Salpêtrière, 47 Boulevard de I'hôpital, F-75013, Paris, France. E-mail: severine.mahon@upmc.fr.

DOI:10.1523/JNEUROSCI.0415-12.2012

Copyright $\odot 2012$ the authors $\quad 0270-6474 / 12 / 3211377-13 \$ 15.00 / 0$
Cortical neurons may also undergo experience-dependent modifications in their intrinsic excitability through modulation of voltage-gated ion channels (Daoudal and Debanne, 2003). By enhancing or depressing the responsiveness of individual neurons to incoming inputs, intrinsic plasticity can strongly affect the collective activity in neural networks (Marder and Prinz, 2002). Changes in cortical neuron excitability have been observed from ex vivo slices following learning procedures (Saar et al., 1998; Matthews et al., 2008) or after experimental manipulation of the levels of activity in vitro and in vivo (Desai et al., 1999; Sourdet et al., 2003; Cudmore and Turrigiano, 2004; Paz et al., 2009). Until now, intrinsic plasticity in barrel cortex neurons has been mainly studied in the context of brain maturation. Whisker trimming during the critical period has been shown to increase the dendritic excitability of layer 5 neurons (Breton and Stuart, 2009), delay the maturation of spiking properties of layer $2 / 3$ cells (Maravall et al., 2004), and decrease cellular excitability of layer 4 fast-spiking interneurons (Sun, 2009). However, an ex vivo study has recently demonstrated that intrinsic excitability of excitatory layer 4 cells could be enhanced following classical conditioning (Bekisz et al., 2010), suggesting that intrinsic plasticity could contribute to learning-related changes in somatosensory representation.

Although there is growing evidence that barrel cortex neurons can display activity-dependent changes in membrane excitability, induction and expression properties of intrinsic plasticity during sensory experience in the adult remain largely unexplored. Exploratory behavior in rodents involves rhythmic sweeping movements of their whiskers over objects at a frequency range of 5-15 $\mathrm{Hz}$ (Carvell and Simons, 1990; Fee et al., 1997). This "whisking 
behavior" is associated with membrane potential oscillations and recurrent action potential (AP) firing in cortical cells (Fee et al., 1997; Ganguly and Kleinfeld, 2004; Crochet and Petersen, 2006). Here, we hypothesize that periods of repeated firing at "whisking frequency" in cortical neurons might be a pertinent paradigm to trigger intrinsic plasticity. We show that periods of currentinduced firing at $10 \mathrm{~Hz}$ trigger in vivo a long-lasting increase or decrease in intrinsic excitability of barrel cortex layer 5 pyramidal neurons, leading to consistent bidirectional changes in whiskerevoked neuronal responses.

\section{Materials and Methods}

The care and experimental manipulation of the animals were performed in accordance with the regulation of the Institut National de la Santé Et de la Recherche Médicale and followed the European Union guidelines (directive 86/609/EEC).

Animals and in vivo electrophysiology. Two- to three-month-old male Sprague Dawley (Charles River) rats $(n=33)$ were initially anesthetized by intraperitoneal injection of sodium pentobarbital $(40 \mathrm{mg} / \mathrm{kg}$; CEVA Santé Animale) and ketamine (50 mg/kg; Imalgène). The animals were then cannulated after incision of the trachea and placed in a stereotaxic frame. Incision and pressure points were repeatedly infiltrated with lidocaine (2\%; CEVA Santé Animale). A small craniotomy was made above the barrel field of the primary somatosensory cortex [S1BF; $6.5-7.6 \mathrm{~mm}$ anterior to the interaural line, $4.5-5.5 \mathrm{~mm}$ lateral to the midline (Paxinos and Watson, 1986)], allowing combined intracellular and electrocorticographic (ECoG) recordings. ECoG activity and heart rate were monitored throughout the experiment to assess the level of anesthesia, and additional doses of pentobarbital $(10-15 \mathrm{mg} / \mathrm{kg})$ were administered as needed. In an additional set of experiments $(n=6)$, rats were maintained in a sedated and analgesied state using injections of fentanyl $(3 \mu \mathrm{g} / \mathrm{kg}$, i.p.; Janssen-Cilag) repeated every $20-30 \mathrm{~min}$, as previously described (Mahon et al., 2001; Polack and Charpier, 2006). To obtain long-lasting stable intracellular recordings, rats were immobilized following intramuscular injection of gallamine triethiodide $(40 \mathrm{mg} / 2 \mathrm{~h}$; Specia) and artificially ventilated.

Spontaneous ECoG activity and sensory-evoked potentials (SEPs) were recorded using a low impedance $(\sim 60 \mathrm{k} \Omega)$ silver electrode placed on the dura above the barrel cortex and a reference electrode inserted in a muscle at the opposite side of the head. Surface cortical signals were amplified by a differential AC amplifier (Model 1700; A-M Systems), filtered at $1 \mathrm{~Hz}-10 \mathrm{kHz}$, and digitized at $1-3 \mathrm{kHz}$ (CED 1401plus; Cambridge Electronic Design). Intracellular recordings were made near $(<300 \mu \mathrm{m})$ the ECoG electrode using glass micropipettes filled with $2 \mathrm{M}$ potassium acetate (50-70 M $\Omega$ ). Current-clamp recordings were amplified using an Axoclamp 2B amplifier (Molecular Devices) in bridge mode, filtered at $3 \mathrm{kHz}$, and digitized at $15-20 \mathrm{kHz}$. Recordings were obtained from layer 5 pyramidal neurons located at depths ranging from 950-1900 $\mu \mathrm{m}$ below the cortical surface (Paxinos and Watson, 1986; Wilent and Contreras, 2004). No holding current was injected during intracellular recordings. All data were acquired and analyzed using Spike2 software (Spike2 version 6.01; Cambridge Electronic Design). Additional data analysis was performed using Origin 7.0 (OriginLab Corporation).

Neuron staining. Neurons were labeled after intracellular injection of $1 \%$ neurobiotin (Vector Laboratories) added to the pipette solution. Positive current pulses ( $0.5-1 \mathrm{nA} ; 100-200 \mathrm{~ms}$ duration) were applied at $1-2 \mathrm{~Hz}$ for $10-15 \mathrm{~min}$ to obtain a reliable labeling of neuronal processes. At the end of experiments, rats were deeply anesthetized with pentobarbital $(200 \mathrm{mg} / \mathrm{kg})$ and transcardially perfused with $0.3 \%$ glutaraldehyde- $4 \%$ paraformaldehyde in PBS (0.1 M, pH 7.4). The morphology of stained neurons was revealed histochemically as described previously (Polack and Charpier, 2006). All neurons recovered were located in layer 5 of the barrel cortex (Paxinos and Watson, 1986) and possessed the typical morphological features of either layer $5 \mathrm{a}$ or $5 \mathrm{~b}$ pyramidal neurons (Schubert et al., 2001, 2006).

Sensory stimulation. Sensory responses were evoked by air-puffs (50 $\mathrm{ms}, 6-40 \mathrm{psi}$ ) timed by a pressure device (Picospritzer III, Intracel) and delivered to the contralateral whiskers. Air-puff stimuli, which deflected four to eight whiskers by $\sim 10$ degrees, were applied $40-100$ times every $3.25-5.25 \mathrm{~s}$ in each control and test periods. Multiwhisker stimulations were preferred to single whisker stimulations as they are more likely to occur during the exploration of an object, even if the number of whiskers involved may vary with behavior (Carvell and Simons, 1990; Kleinfeld et al., 2006; Fox, 2008). At the beginning of each experiment, the orientation and intensity of the air puff were adjusted to generate the SEP of maximal amplitude in the contralateral barrel cortex.

Assessment of intrinsic excitability and conditioning protocols. To generate firing frequency versus injected current $(F-I)$ relationships, the firing rate was measured in response to depolarizing current pulses of increasing intensity (500 ms, $0.1-1.4 \mathrm{nA}$ ) delivered with an interstimulus interval of 3.25-5.25 s. Each current intensity was applied 20-25 times and the corresponding firing responses were averaged. As previously described (Paz et al., 2009), we applied linear regressions to $F-I$ curves and determined the threshold current for AP generation, extrapolated as the $x$-intercept of the linear fit (Fig. 1D), and the neuronal gain, defined as the slope of the $F-I$ curve. Intrinsic plasticity was induced by trains of 10 depolarizing current pulses (50 $\mathrm{ms})$ at $10 \mathrm{~Hz}$ repeated every $3 \mathrm{~s}$ during $5-7 \mathrm{~min}(5.80 \pm 0.15 \mathrm{~min}, n=$ 30 neurons). The intensity of the conditioning stimulus (0.3-1.4 nA) was adjusted to evoke $1-3 \mathrm{APs}$ on each current pulse (mean $=2.4 \pm$ 0.2 APs, $n=30$ ) (Fig. $1 B$ ). The mean firing rate during induction, which included the current-evoked spikes and the spontaneous discharge in between trains, was of $10.1 \pm 0.9 \mathrm{~Hz}(n=30)$. The frequency of stimulations within a train and the delay between trains were designed to approximate whisking frequency and periodicity of "whisking episodes," as reported in awake rodents (Carvell and Simons, 1990; Ganguly and Kleinfeld, 2004; Crochet and Petersen, 2006).

Data analysis. In addition to changes in $F-I$ curves, modifications in intrinsic excitability in response to depolarizing current pulses were assessed by measuring, for a given stimulus intensity, firing rate, first spike latency, first spike voltage threshold, and the slope of membrane depolarization to the first spike. Voltage spike threshold was defined as the membrane potential at which $d V / d t$ first exceeds $10 \mathrm{~V} / \mathrm{s}$ (Fricker et al., 1999; Mahon et al., 2003). The slope of membrane depolarization preceding the first spike was calculated over a period of 10-15 ms before spike threshold and approximates a linear voltage trajectory.

The spontaneous firing rate of cortical neurons was measured for periods of 1-2 min before and after the conditioning. Average membrane potential $\left(V_{\mathrm{m}}\right)$ values were calculated as the mean of the distribution of spontaneous intracellular subthreshold activity recorded for 10-60 s. When necessary, they were corrected by subtracting the extracellular tip potential measured immediately after the loss of the recording. Membrane input resistance $\left(R_{\text {in }}\right)$ was calculated, during control and postinduction periods, from averaged $(n \geq 10)$ voltage deflections during hyperpolarizing current pulse injection $(-0.4 \mathrm{nA}, 200 \mathrm{~ms}$ duration, applied every $1.25 \mathrm{~s}$ ). The membrane time constant was derived from an exponential decay fit applied to the current-evoked hyperpolarization. Cross-correlations between ECoG signal and subthreshold intracellular activity were performed after down-sampling the two signals at $1 \mathrm{kHz}$. The dominant frequency of ECoG potentials was calculated by applying fast Fourier transforms to segments (10-60 s) of the ECoG record using the Power Spectrum tool in Spike2.

Latency of sensory-evoked intracellular responses was calculated as the time between the onset of the air-puff stimulus and the start of the cell response. Layer 5 pyramidal neurons in vivo may exhibit some variability in their responses to sensory stimulation, likely due to interactions with ongoing spontaneous activity (Petersen et al., 2003; Sachdev et al., 2004). Only individual responses with latency and rising phase matching those of the mean evoked-response (averaging of 30-50 successive trials) were considered for analysis. Typically, $\sim 5-10 \%$ of responses were discarded. Amplitude of subthreshold postsynaptic potentials (PSPs) was measured from the foot to the peak of the synaptic depolarization. The rising slope of sensory responses was determined by approximating the membrane depolarization, from the onset of the PSP to the spike threshold or to the 

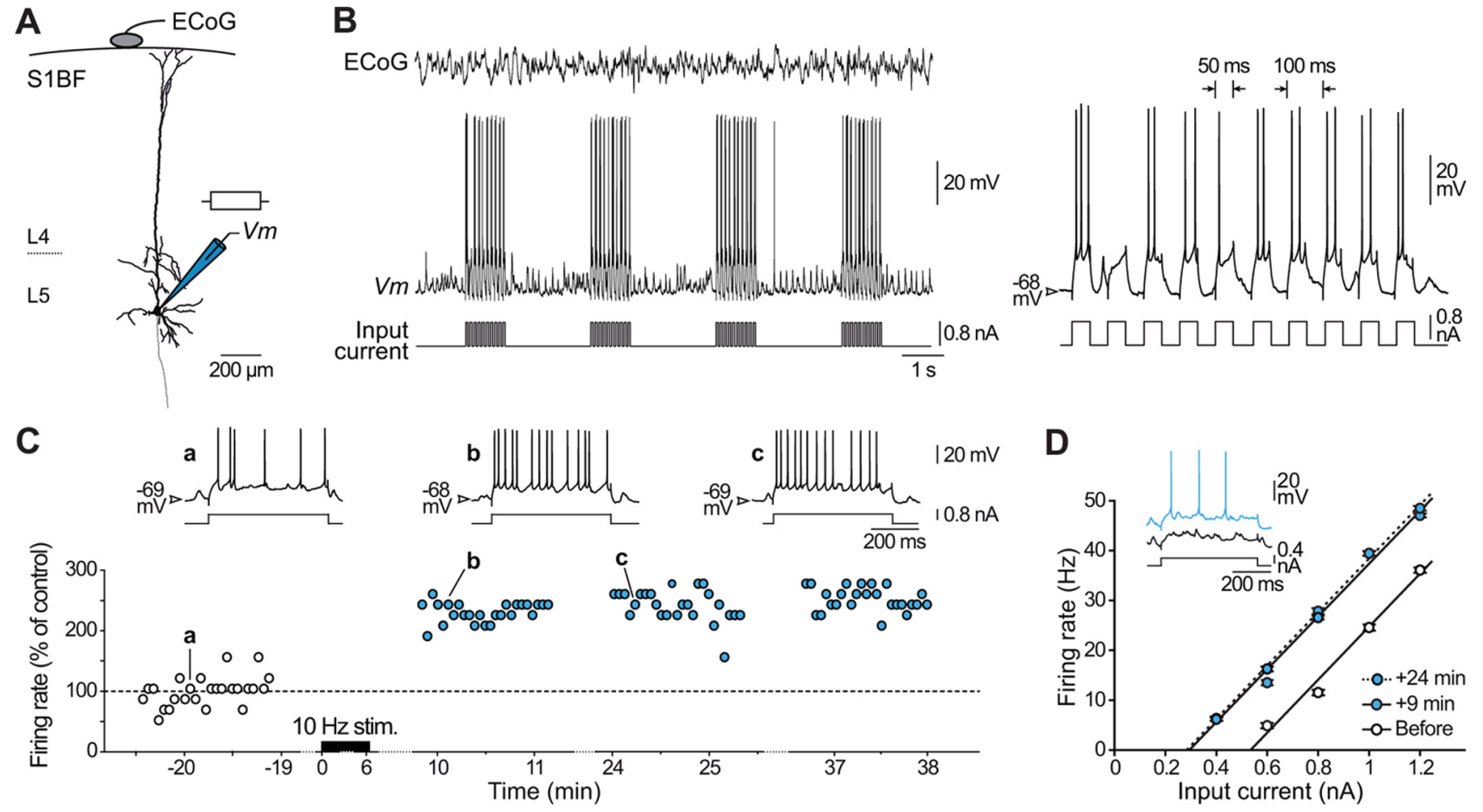

E

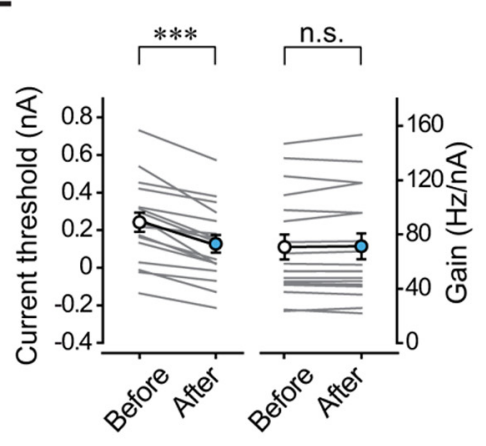

G

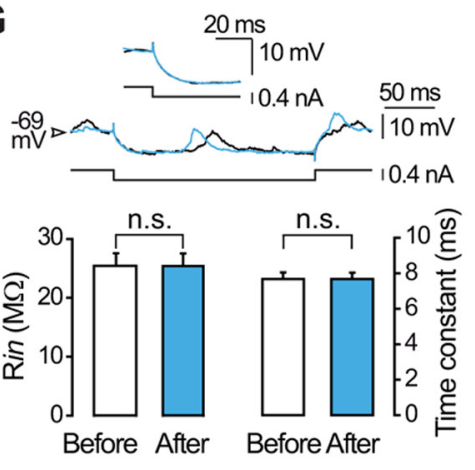

F1

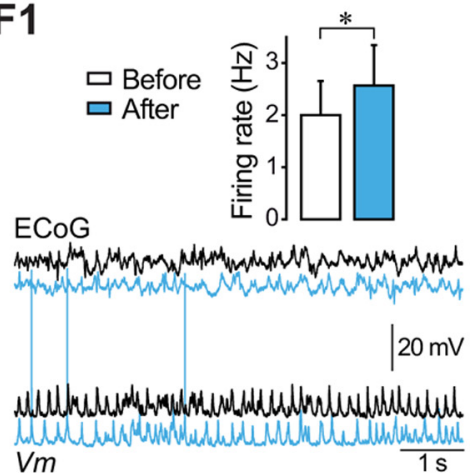

F2

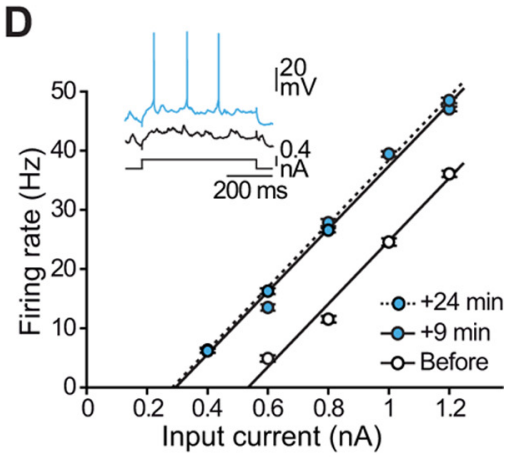

H

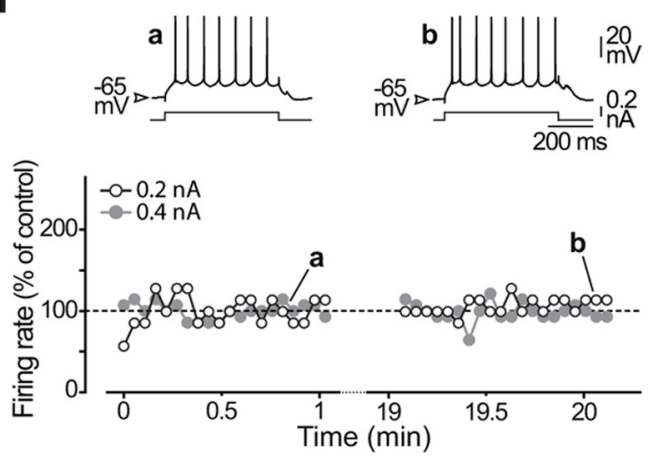

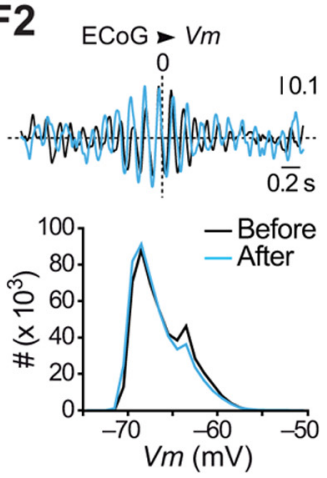
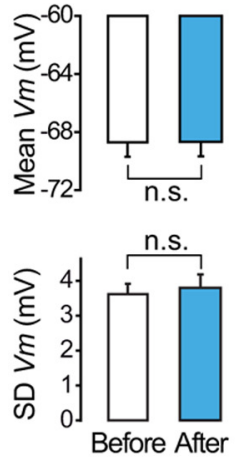

Figure 1. Activity-dependent long-lasting potentiation of intrinsic excitability in layer 5 pyramidal neurons in vivo. $A$, Reconstruction of a layer 5 pyramidal neuron of the rat barrel cortex (S1BF) filled with neurobiotin and recording configuration. ECoG was monitored during intracellular recording of $V_{\mathrm{m}}$ and intrinsic excitability was assessed in response to depolarizing and hyperpolarizing current pulses injected via the microelectrode. $\boldsymbol{B}$, Simultaneous ECOG and intracellular recordings of a layer 5 pyramidal neuron during a portion of the conditioning protocol, which consisted in the injection of trains of suprathreshold current pulses every 3 for $5-7$ min. Right, Expanded record (first train in the left panel) showing that each train of stimulations was composed by 10 depolarizing current pulses of $50 \mathrm{~ms}$ duration applied at $10 \mathrm{~Hz}$. C, Time course and magnitude of LLPIE in a representative potentiated neuron. Firing rate versus time evoked by a current pulse of constant amplitude ( $+0.8 \mathrm{nA})$, in control (white symbols) and after (blue symbols) application of the $10 \mathrm{~Hz}$ conditioning stimuli ( $10 \mathrm{~Hz}$ stim.). Changes in cell discharge are normalized to the mean control value (dashed line). The upper traces illustrate the current-evoked responses at the indicated times. Here and in subsequent figures, membrane potential values are indicated at the left of the records. $D, F-/$ relationships and corresponding linear fits before and at the indicated times after induction. Each data point is the mean ( \pm SEM) firing rate calculated from $25 \mathrm{successive} \mathrm{trials} \mathrm{(every} 3.25 \mathrm{~s}$ ). The current threshold was $0.54 \mathrm{nA}$ before conditioning and decreased to $0.29 \mathrm{nA} 24$ min after induction. Inset, Single records showing that a previously subthreshold stimulus (black trace) became suprathreshold (blue trace) after induction. $\boldsymbol{E}$, Summary data ( $n=18$ neurons) showing a significant decrease in current threshold values after conditioning while the neuronal gain remained (Figure legend continues.) 
peak of subthreshold PSP, as a straight line. Firing probability was estimated as the ratio between the number of suprathreshold responses and the total number of evoked-responses. Latency of evoked spikes on suprathreshold PSPs was the time between the onset of the sensory stimulus and the peak of the spike waveform.

Numerical values are given as mean \pm SEM for the number of neurons indicated. Statistical significance was assessed using paired and unpaired two-tailed Student's $t$ tests, one-way ANOVA, or the non-parametric Wilcoxon signed rank test, Mann-Whitney rank sum test, and KruskalWallis one-way ANOVA on Ranks when appropriate (SigmaStat version 3.5, Systat Software).

\section{Results}

\section{Activity-dependent bidirectional changes in intrinsic excitability}

We obtained in vivo intracellular recordings from pyramidal neurons located in layer 5 of the barrel cortex $(n=42)$ together with the corresponding EcoG (Fig. 1 $A, B$ ). To determine whether periods of repeated firing at whisking frequency can trigger changes in cortical excitability, pyramidal neurons $(n=30)$ were challenged with trains of suprathreshold current pulses at $10 \mathrm{~Hz}$ delivered every $3 \mathrm{~s}$ during 5-7 min (Fig. $1 B)$. Neuronal excitability was assessed by measuring the firing rate in response to $500 \mathrm{~ms}$ depolarizing current pulses in control and at different times after conditioning. We found that the induction protocol could produce an increase in current-evoked firing in some barrel cortex neurons and a decrease in others. Across the population, potentiation or depression of intrinsic excitability was observed in $60 \%$ (18 of 30 ) and $40 \%$ (12 of 30 ) of the conditioned cells, respectively.

In the group of potentiated neurons, the increase in currentinduced spiking was already present a few minutes after the conditioning and persisted throughout the recording (Fig. 1C), indicating that short periods of $10 \mathrm{~Hz}$ firing caused long-lasting potentiation of intrinsic excitability (LLPIE) in pyramidal cells (up to $48 \mathrm{~min}$ after conditioning). Across the population ( $n=$ 18 ), average firing rates measured 20-30 min after induction were significantly higher than those preceding conditioning $(p<$ 0.001 ) and amounted to $164.9 \pm 15.2 \%$ of control values (Fig. $1 C)$. To assess whether the increase in excitability could be expressed over a range of input currents, we next generated $F-I$ curves during baseline and postconditioning periods. The num-

\footnotetext{
$\leftarrow$

(Figure legend continued.) unchanged. Here and in similar figures, gray lines indicate values for individual experiments and white/blue symbols show mean values \pm SEM. $\boldsymbol{F}$, Impact of LLPIE on spontaneous activity. F1, Typical recordings of spontaneous intracellular activity and corresponding $\mathrm{EC} 0 \mathrm{G}$ waves, before (black traces) and after plasticity induction (blue traces). The inset shows average values ( $n=18$ neurons) of spontaneous firing rates before and during LLPIE. F2, Left, Corresponding cross-correlograms between EC $G$ and intracellular signals (computed from 10 s of recording; top) and $V_{\mathrm{m}}$ distributions ( 60 s of recording, bin size $1 \mathrm{mV}$; bottom) showing the stability of intensity and frequency of synaptic activities over time. Right, Pooled data ( $n=18$ neurons) comparing the mean and SD of $V_{m}$ values in control and postinduction periods. $\mathbf{G}$, As illustrated by the neuron responses to hyperpolarizing pulses of $0.4 \mathrm{nA}$ (upper traces) in control (black trace) and during LLPIE (blue trace), membrane time constant and $R_{\text {in }}$ were not significantly modified by the conditioning (bottom graphs). The inset is an expansion of the onset of voltage responses. $\boldsymbol{H}$, Stability of intrinsic excitability in unconditioned neurons. Left, Time course of firing rate evoked by depolarizing current pulses of $0.2 \mathrm{nA}$ (white symbols) and $0.4 \mathrm{nA}$ (gray symbols) in a cortical neuron that was not challenged with the conditioning pulse-trains. The mean firing rates (normalized to the mean value of the first control period, dashed line) remained stable during the two control periods ( $p>0.2$ for each intensity tested). Examples of current-induced firing responses, recorded at the indicated times, are shown above the graph. Right, Population data ( $n=9$ neurons) showing the constancy of current threshold and gain values in control recordings. ${ }^{*} p<0.05 ;{ }^{* * *} p<0.001 ;$ n.S., nonsignificant. $\boldsymbol{B}-\boldsymbol{D}$ and $\boldsymbol{F}$ all relate to the same neuron.
}

ber of APs elicited by any current intensity was increased after induction $(p<0.001)$, leading to a leftward shift in $F-I$ curves (Fig. 1D). In each neuron, the current threshold for evoking spikes was found to be significantly reduced, from $0.24 \pm 0.05 \mathrm{nA}$ in control to $0.13 \pm 0.05 \mathrm{nA}$ after conditioning $(n=18 ; p<$ 0.001 ), while the neuronal gain, measured as the slope of the linear portion of $F-I$ curves, remained unaffected by the conditioning (before, $70.8 \pm 9.2 \mathrm{~Hz} / \mathrm{nA}$ vs after, $71.3 \pm 9.4 \mathrm{~Hz} / \mathrm{nA}, n=$ $18 ; p>0.5$ ) (Fig. $1 D, E)$.

Neurons experiencing LLPIE exhibited a moderate but significant increase in spontaneous firing (before, $2.0 \pm 0.6 \mathrm{~Hz}$ vs after, $2.6 \pm 0.8 \mathrm{~Hz}, n=18 ; p<0.05$ ) (Fig. 1 F1). To ascertain that the synaptic drive to potentiated neurons remained stable during the course of the recordings, we compared the amplitude of spontaneous $V_{\mathrm{m}}$ fluctuations and the pattern of ECoG waves before and following LLPIE induction (Fig. $1 F 1, F 2$ ). The overlaid distributions of $V_{\mathrm{m}}$ values (Fig. 1 F2, left) demonstrated that mean amplitude $(n=18 ; p>0.6)$ and fluctuations $(n=18 ; p>0.1)$ of spontaneous synaptic events were not affected by the conditioning (Fig. 1 F2, right). The dominant frequency of ECoG waves was also found unchanged after conditioning (before, $6.8 \pm 0.3 \mathrm{~Hz}$ vs after, $6.7 \pm 0.3 \mathrm{~Hz}, n=18 ; p>0.5)$. Consistently, the sign and phase of the correlation between intracellular and ECoG activities were similar after plasticity induction compared with control period, indicating that the level of synaptic activity in the local neural network remained stable for the duration of the recording (Fig. 1F2). $R_{\text {in }}$ and membrane time constant were not significantly modified after induction $(<1 \%$ change on average compared with control, $n=18 ; p>0.8$ for both parameters), demonstrating that LLPIE was not associated with any alteration in the passive membrane properties (Fig. $1 G$ ).

In control neurons $(n=9)$, recorded for long periods without delivering conditioning trains, the number of APs elicited by any given current pulse remained stable for the duration of the recording (Fig. $1 \mathrm{H}$, left), indicating the specific effect of the induction protocol. When successive $F-I$ curves were constructed in these unconditioned neurons, we did not detect changes in current threshold (Ctrl1, $0.13 \pm 0.06 \mathrm{nA}, n=9$ vs Ctrl2, $0.12 \pm 0.05$ $\mathrm{nA}, n=9 ; p>0.3)$ and neuronal gain $(\mathrm{Ctrl1}, 64.4 \pm 11.1 \mathrm{~Hz} / \mathrm{nA}$, $n=9$ vs Ctrl2, $64.4 \pm 11.3 \mathrm{~Hz} / \mathrm{nA}, n=9 ; p>0.9$ ) over time (Fig. $1 \mathrm{H}$, right). To ascertain that the anesthetic procedure used in this study did not significantly affect the membrane excitability of cortical cells, we performed an additional set of experiments in rats $(n=6)$ sedated and analgesied with fentanyl, which induces a relatively desynchronized ECoG pattern resembling that observed during wakefulness (Mahon et al., 2001; Constantinople and Bruno, 2011). The average baseline values of $V_{\mathrm{m}}(-65.5 \pm$ $1.3 \mathrm{mV}, n=11$ neurons), $R_{\text {in }}(31.8 \pm 2.8 \mathrm{mV}, n=11)$, current threshold $(0.10 \pm 0.07 \mathrm{nA}, n=11)$, and gain $(77.4 \pm 10.0 \mathrm{~Hz} / \mathrm{nA}$, $n=11)$ in cortical neurons under fentanyl were similar $(p>0.4$ for each parameter) to those measured in control cells recorded under barbiturate anesthesia $(n=9)$.

In the second group of conditioned neurons $(n=12)$, the $10 \mathrm{~Hz}$ pulse trains resulted in a long-lasting depression in intrinsic excitability (LLDIE). As illustrated in Figure $2 \mathrm{~A}$, the decrease in excitability was evident over the full range of tested currents and persisted throughout the postconditioning period, with average firing rate reduced by $35.7 \pm 4.5 \%(n=12$; $p<0.001$ ) 20-30 min after induction (Fig. 2A1,A2). Depression of intrinsic excitability was associated with a significant increase in current threshold values (before, $-0.02 \pm 0.04 \mathrm{nA}$ vs after, $0.07 \pm 0.04 \mathrm{nA}, n=12 ; p<0.01$ ) (Fig. $2 B$ ), leading to a rightward shift of $F-I$ curves without any substantial change 
A1
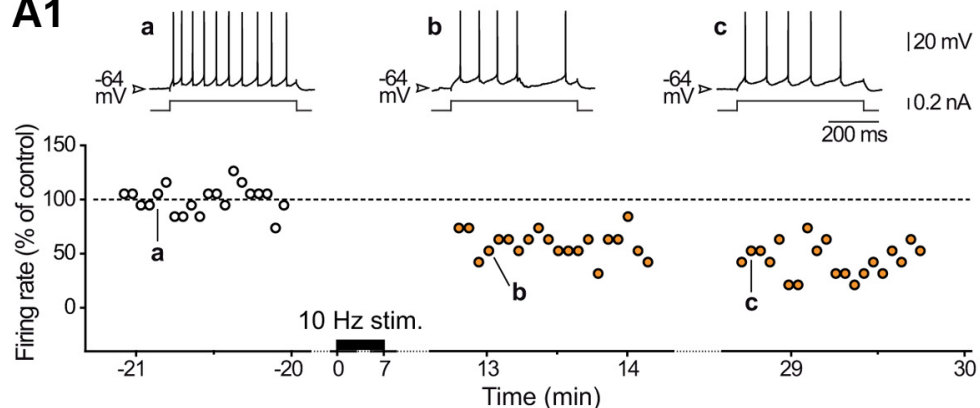

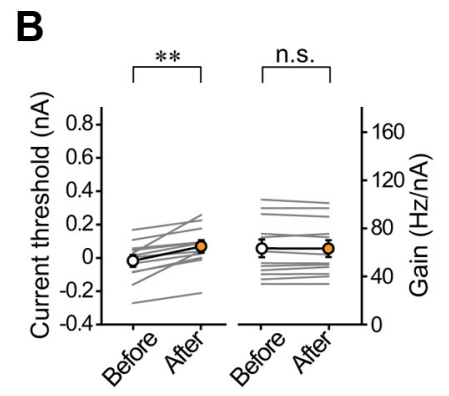

C
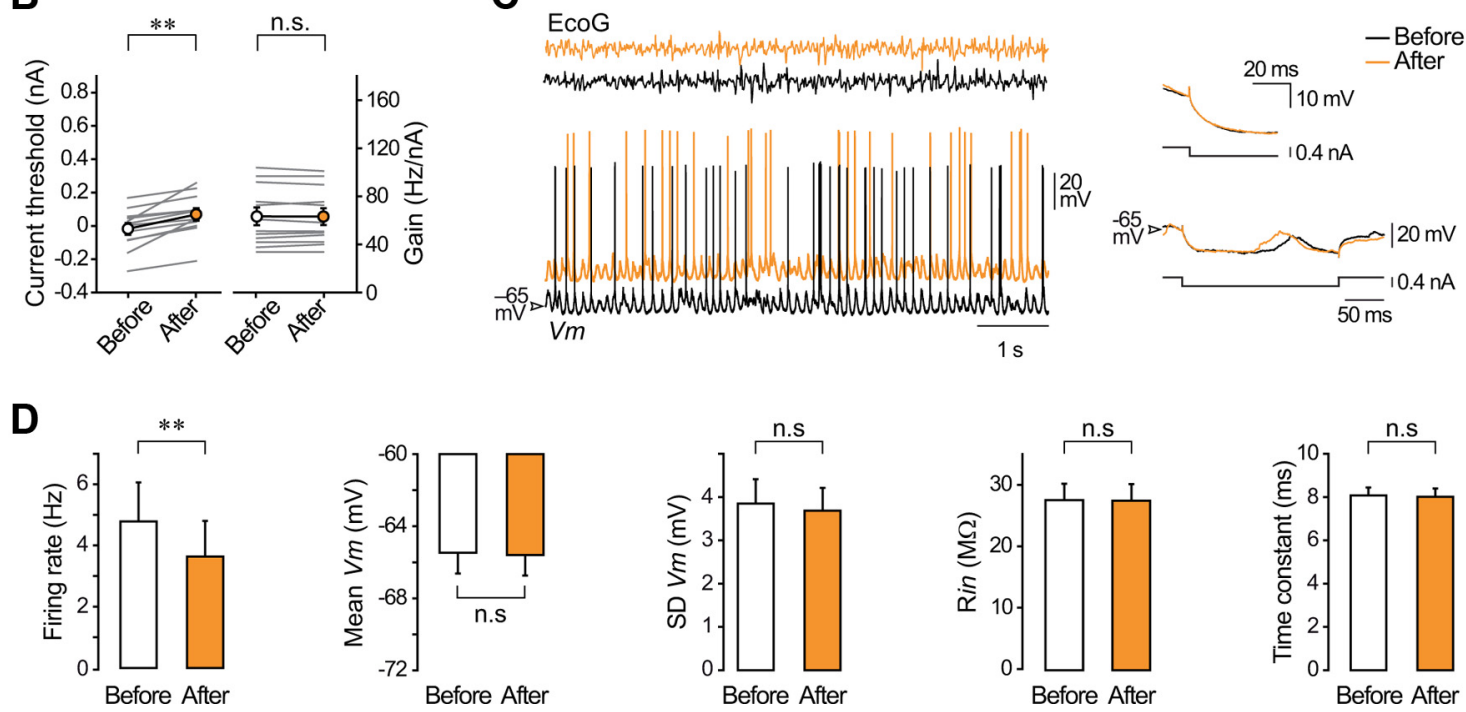

Figure 2. Long-lasting depression of intrinsic excitability in layer 5 pyramidal neurons in vivo. A, Time course and magnitude of plasticity in a barrel cortex neuron exhibiting LLDIE. A1, Long-lasting decrease in firing frequency in response to a depolarizing pulse of constant amplitude $(0.2 \mathrm{nA})$ following a 7 min period of conditioning $(10 \mathrm{~Hz}$ stim.). Changes in firing rate are expressed in percentage of the mean control value (dashed line). Evoked responses, obtained at the indicated times before and after conditioning, are shown on top. $A 2$, Corresponding $F-I$ curves constructed in control and after induction at the indicated times. Each symbol corresponds to the mean ( \pm SEM) firing rate calculated from 20 successive (every $4.25 \mathrm{~s}$ ) trials. Note the decrease in current threshold, from $0.013 \mathrm{nA}$ to $0.09 \mathrm{nA} 28 \mathrm{~min}$ after induction, without modification in the slope of $F-I$ curves. Suprathreshold responses to depolarizing current injection of $0.1 \mathrm{nA}$ could become subthreshold after conditioning (inset). $\boldsymbol{B}$, Population analysis ( $n=12$ neurons) of current threshold and gain values before and after LLDIE induction. Gray lines show values for individual experiments and white/orange symbols indicate the corresponding mean values \pm SEM, here and in similar figures. $C$, Left, Epochs of spontaneous intracellular and ECoG activities before (black traces) and after plasticity induction (orange traces). Right, Superimposition of individual and mean (12 successive trials, inset) voltage responses to negative current pulses delivered before and after conditioning. $\boldsymbol{D}$, Summary data ( $n=12$ neurons) comparing average values of spontaneous firing rate, mean, and SD of $V_{\mathrm{m}}$, $R_{\mathrm{in}}$, and membrane time constant from control and postinduction periods. ${ }^{* *} p<0.01 ;$ i.s., nonsignificant.

in the neuronal gain (before, $63.3 \pm 7.3 \mathrm{~Hz} / \mathrm{nA}$ vs after, $63.2 \pm$ $7.0 \mathrm{~Hz} / \mathrm{nA}, n=12 ; p>0.8$ ) (Fig. $2 A 2, B$ ). The induction procedure significantly reduced the spontaneous firing of neurons that showed LLDIE $(24.6 \pm 8.8 \%$ decrease, $n=12$; $p<0.01$ ) (Fig. $2 C, D)$. Again, $R_{\text {in }}(n=12 ; p>0.4)$ and membrane time constant $(n=12 ; p>0.8)$ remained unaffected by the conditioning (Fig. 2C,D). As observed during LLPIE, the dominant frequency of ECoG waves (before, $6.5 \pm 0.4 \mathrm{~Hz}$ vs after, $6.6 \pm 0.4 \mathrm{~Hz}, n=12 ; p>0.3$ ) as well as mean value, amplitude, and frequency of $V_{\mathrm{m}}$ fluctuations of depressed neurons remained stable over time $(n=12 ; p>0.2)$ (Fig. $2 C, D)$.

Conditioned neurons exhibited either a regular spiking pattern (RS, $n=23$; Figs. $1 C, 2 A 1$ ) or an intrinsic bursting profile (IB; $n=7$ ) in response to suprathreshold current steps (Zhu and Connors, 1999). Both cell types could undergo potentiation (14 RS and 4 IB) or depression (9 RS and 3 IB) of membrane excitability, without switching of the firing mode after conditioning. In addition, LLPIE and LLDIE could be obtained in the same preparation $(n=3)$, indicating that the direction of plasticity was not animal dependent.
Our results indicate that a few minutes of cell firing at $10 \mathrm{~Hz}$ leads to a long-lasting potentiation of intrinsic excitability in some layer 5 barrel cortex neurons and a depression of excitability in others. These opposing plasticities were characterized by antagonistic changes in the threshold current for AP generation without modification in neuronal gain.

\section{Bidirectional intrinsic plasticity differentially affects firing properties}

We further characterized intrinsic plasticity by comparing the temporal properties of current-evoked firing and voltage threshold for AP discharge before and after conditioning. Measurements of AP voltage threshold were restricted to RS neurons because of the large depolarization underlying burst firing in IB cells.

We first compared the latency and temporal precision of the first evoked spike in neurons expressing LLPIE. Precision of AP discharge was quantified as spike jitter defined as the SD of the first AP latency across successive trials. In addition to increasing firing rate, the induction protocol produced a decrease in the first spike latency (before, $48.0 \pm 8.6 \mathrm{~ms}$ vs after, $28.3 \pm 5.4 \mathrm{~ms}, n=$ 
A

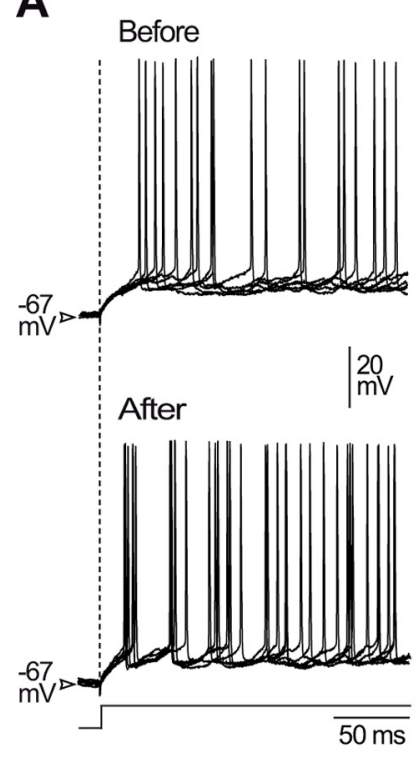

D
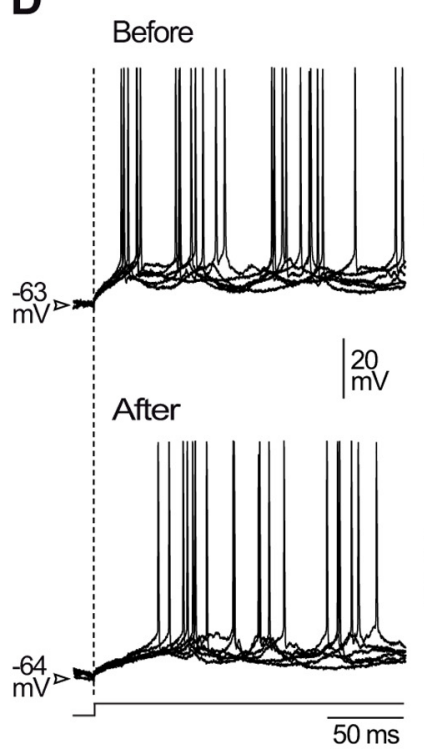

1st spike
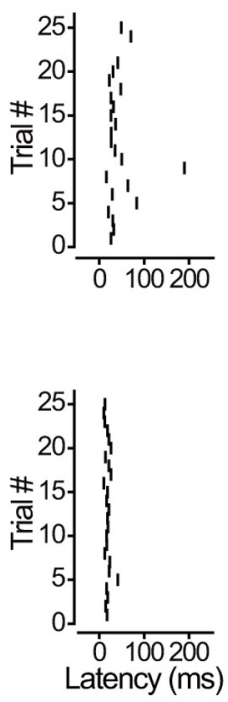

Latency (ms)
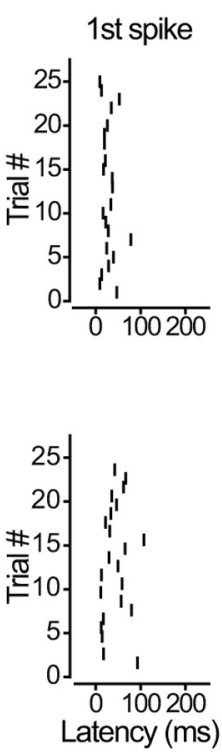

B
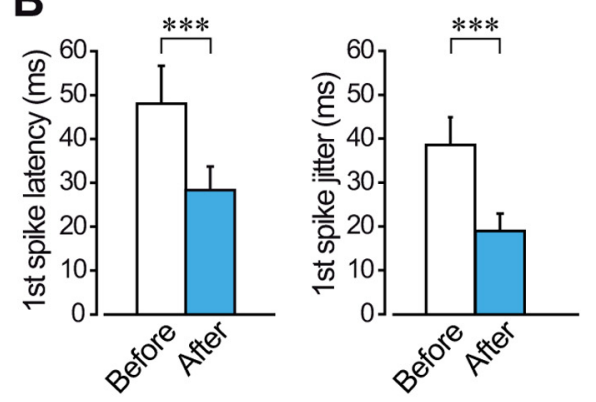

C
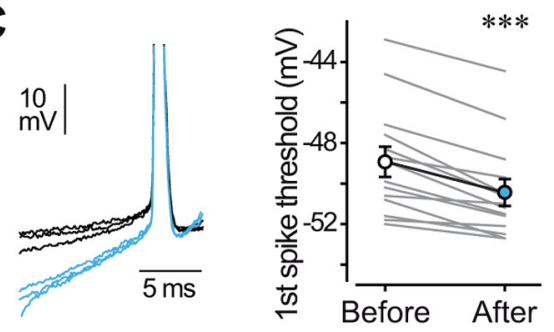

E
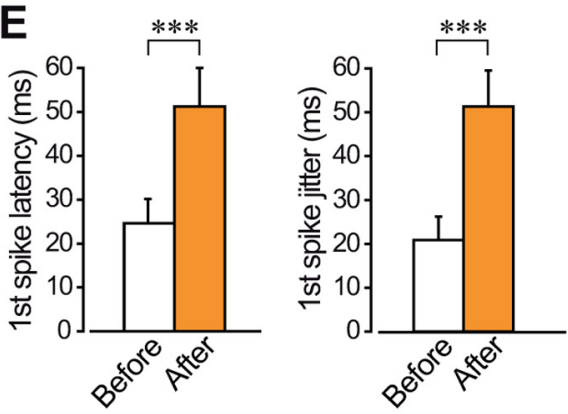

$\mathbf{F}$
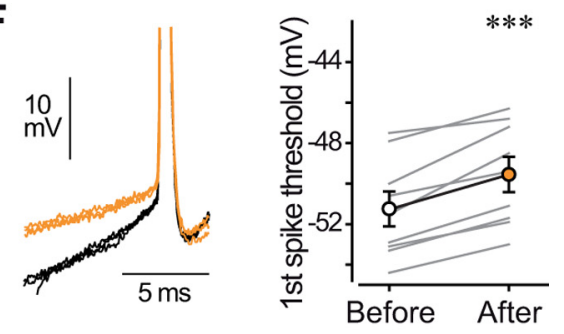

Figure 3. Long-lasting intrinsic plasticity alters firing properties. A, Six overlaid firing responses (the first $200 \mathrm{~ms}$ are illustrated) of a barrel cortex neuron to injection of current pulses ( $+0.4 \mathrm{nA}$, $500 \mathrm{~ms}$ ) before (top traces) and during (bottom traces) LLPIE. The dashed line indicates the onset of the current injection. The corresponding raster plots of the first spikes (25 successive trials) are shown at right. $B$, Mean values of first spike latency and jitter before and after LLPIE induction ( $n=18$ neurons). $C$, DC superimposition of the first spikes evoked by current injection in control (black traces, $n=3$ ) and after conditioning (blue traces, $n=3$ ) showing that LLPIE was associated with an increase in the slope of membrane depolarization preceding the first action potential and with a lowered voltage spike threshold. Spikes are from records in $A$ and truncated for convenience. Population analysis (right, $n=14$ RS neurons) reveals a postconditioning decrease in the first spike threshold in all computed cells. D, Six overlaid firing responses of a barrel cortex neuron to injection of $+0.2 \mathrm{nA}$ current pulses before (top traces) and after (bottom traces) LLDIE induction. The corresponding raster plots of the first spikes (22 successive trials) are shown at right. $\boldsymbol{E}$, Mean values of first spike latency and jitter before and after LLDIE induction ( $n=12$ neurons). $\boldsymbol{F}$, DC superimposition of the first spikes evoked by current injection in control (black traces, $n=3$ ) and after conditioning (orange traces, $n=3$ ) showing that LLDIE was correlated with a decrease in the slope of the prespike voltage trajectory and with an augmented voltage spike threshold. Same cell as in $\boldsymbol{D}$ is used. Population analysis (right, $n=9 \mathrm{RS}$ neurons) reveals a significant increase in the first spike threshold after induction. ${ }^{* * *} p<0.001$.

$18 ; p<0.001$ ) and a reduced first spike jitter (before, $38.6 \pm 6.4$ ms vs after, $19.0 \pm 3.9 \mathrm{~ms}, n=18 ; p<0.001$ ) (Fig. $3 A, B$ ). LLPIE induction was also accompanied by a lowering of the first spike voltage threshold (before, $-48.9 \pm 0.7 \mathrm{mV}$ vs after, $-50.4 \pm 0.7$ $\mathrm{mV}, n=14$ RS neurons; $p<0.001$ ), which was correlated with an increase in the slope of membrane depolarization preceding cell discharge $(1.07 \pm 0.12 \mathrm{mV} / \mathrm{ms}$ in control vs $1.40 \pm 0.16 \mathrm{mV} / \mathrm{ms}$ after conditioning, $n=14 ; p<0.001$ ) (Fig. $3 C$ ).

Opposite changes in firing properties were found in neurons experiencing LLDIE. Depression of intrinsic excitability was ac- companied by an increase in the first spike latency (before, $24.6 \pm$ $5.5 \mathrm{~ms}$ vs after, $51.2 \pm 8.8 \mathrm{~ms}, n=12 ; p<0.001)$ and an enhanced jitter of the first spike (before, $20.9 \pm 5.3 \mathrm{~ms}$ vs after, $51.3 \pm 8.2$ ms, $n=12$; $p<0.001$ ) (Fig. $3 D, E$ ). In addition, the $10 \mathrm{~Hz}$ conditioning resulted in a more positive first spike voltage threshold (before, $-51.2 \pm 0.9 \mathrm{mV}$ vs after, $-49.5 \pm 0.9 \mathrm{mV}, n=$ 9 RS neurons; $p<0.001)$ associated with a slower rate of rise of the preceding membrane depolarization $(1.39 \pm 0.20 \mathrm{mV} / \mathrm{ms}$ in control vs $1.11 \pm 0.17 \mathrm{mV} / \mathrm{ms}$ after conditioning, $n=9 ; p<0.01$ ) (Fig. 3F). 
These results indicate that induction of LLPIE or LLDIE in pyramidal barrel cortex neurons are associated with distinct changes in latency, precision, and voltage threshold of currentevoked APs.

\section{What are the neuronal parameters influencing the sign of intrinsic plasticity?}

Since similar conditioning protocols could induce either LLPIE or LLDIE in barrel cortex neurons, we attempted to determine whether the sign of intrinsic plasticity was related to the baseline electrophysiological properties of conditioned neurons.

The sign of plasticity could not be linked to distinctive passive membrane properties as no differences in $R_{\text {in }}$ and membrane time constant ( $p>0.4$ for both parameters) were found between potentiated and depressed cells (Fig. 4A1,A2).

Cortical neurons experiencing a depression in their intrinsic excitability exhibited on average a higher baseline spontaneous firing rate (LLPIE, $2.0 \pm 0.6 \mathrm{~Hz}, n=18$ vs LLDIE, $4.8 \pm 1.3 \mathrm{~Hz}$, $n=12 ; p<0.05$ ) together with a more depolarized $V_{\mathrm{m}}$ (LLPIE, $-68.7 \pm 1.0 \mathrm{mV}, n=18$ vs LLDIE, $-65.5 \pm 1.1 \mathrm{mV}, n=12 ; p<$ 0.05 ) (Fig. 4 B1,B2). Since the intensity of the conditioning current pulses was adjusted to generate a similar firing rate in each tested cell (see Material and Methods), we found that the ratio between the mean discharge during conditioning and the baseline firing rate was higher in potentiated cells compared with depressed cells (LLPIE, $39.3 \pm 15.2, n=16$ vs LLDIE, $16.1 \pm$ 13.9, $n=12 ; p<0.05)$. The elevated spontaneous discharge in depressed cells during baseline periods could reflect a more intense synaptic drive onto these neurons or a higher level of intrinsic excitability. The amplitude of membrane potential fluctuations $(p>0.5)$ and dominant frequency of ECoG waves (LLPIE, $6.8 \pm 0.3 \mathrm{~Hz}, n=18$ vs LLDIE, $6.5 \pm 0.4 \mathrm{~Hz}$, $n=12 ; p>0.2)$ were not significantly different between neurons exhibiting LLPIE or LLDIE, suggesting an equivalent global amount of synaptic activity impinging on depressed and potentiated neurons. The comparison of control F-I curve parameters indicated that the extrapolated value of threshold current for AP initiation was on average lower in LLDIE neurons than in LLPIE neurons (LLPIE, $0.24 \pm 0.05$ $\mathrm{nA}, n=18$ vs LLDIE, $-0.02 \pm 0.04 \mathrm{nA}, n=12 ; p<0.001)$, whereas the gain of the corresponding input-output relationships was similar $(p>0.7)$ (Fig. $4 C 1, C 2)$. We searched for a correlation between control current threshold values and the baseline spontaneous firing rate or mean $V_{\mathrm{m}}$. We found that firing threshold progressively decreased with increase in firing rate or a depolarization of mean $V_{\mathrm{m}}$ (Fig. $4 D$ ). We further compared between both neuronal groups the firing properties on current-induced responses with similar spike rates. In depressed neurons, the first AP was evoked with a shorter latency (LLPIE, $48.0 \pm 8.6 \mathrm{~ms}, n=18$ vs LLDIE, $24.6 \pm 5.5 \mathrm{~ms}, n=12$; $p<0.05$ ), a higher temporal precision (LLPIE, $38.6 \pm 6.4 \mathrm{~ms}$, $n=18$ vs LLDIE, $20.9 \pm 5.3 \mathrm{~ms}, n=12 ; p<0.05)$, and from a lower voltage threshold (LLPIE, $-48.9 \pm 0.7, n=14$ vs LLDIE, $-51.2 \pm 0.9 \mathrm{mV}, n=9 ; p<0.05$ ) (Fig. 4C1). The duration and amplitude of APs were similar in both groups of neurons $(p>0.2)$. Together, these results indicate that neurons exhibiting LLDIE after induction have a relatively elevated control intrinsic excitability, characterized mainly by a lower threshold current and a more depolarized $V_{\mathrm{m}}$, likely responsible for the higher spontaneous firing rate. Moreover, we found that the sign of plasticity was inversely correlated with the initial value of current threshold (Fig. $4 E$ ), further demonstrating the dependence of plasticity on the baseline cortical excitability.

We also explored the possibility that the two forms of plasticity were expressed by neurons having different morpho-functional features or cortical location. Different classes of layer 5 pyramidal cells have been described according to their morphology, sublaminar localization, and firing profile (Schubert et al., 2001 ; 2006). As determined by the reconstruction of neurobiotin-filled neurons, neurons with thin- and thick-tufted dendritic arborization were present in both groups. In addition, neurons from the two populations exhibited RS or IB firing patterns in response to suprathreshold current steps and the depth of intracellular recordings did not differ between potentiated and depressed cells (LLPIE, $1349.7 \pm 78.7 \mu \mathrm{m}, n=18$ vs LLDIE, $1385.1 \pm 85.8 \mu \mathrm{m}, n=12 ; p>0.7)$.

\section{Bidirectional changes in the efficiency of sensory inputs}

We hypothesized that the use-dependent modifications in intrinsic excitability could provide a cellular mechanism controlling the efficiency of incoming synaptic inputs. We thus applied, during control and postconditioning periods, sensory stimulations consisting in air-puffs delivered to the whiskers and recorded the induced responses in contralateral barrel cortex neurons simultaneously with the corresponding surface SEPs (Fig. 5A). In control conditions, sensory stimuli $(17.9 \pm 2.6$ psi $)$ generated PSPs that induced firing with a wide range of probabilities (range: $0.06-0.89$, mean $=0.36 \pm 0.07, n=19)$. Consistent with previous findings (Sachdev et al., 2004; Wilent and Contreras, 2004; Pidoux et al., 2011), the average amplitude of whisker-evoked subthreshold PSPs was $12.2 \pm 0.8 \mathrm{mV}(n=19)$ with an onset and a peak latency of $16.1 \pm 0.5 \mathrm{~ms}$ and $22.3 \pm 0.7 \mathrm{~ms}(n=19)$, respectively. Before applying the conditioning procedure, we tested the stability of sensory responses by performing successive control series of air-puff stimuli ( $n=9$ neurons) (Fig. $5 B$ ). As illustrated in Figure 5B1, amplitude of subthreshold PSPs, firing probability, and membrane potential value measured at the foot of synaptic responses remained stable throughout the recording session ( $n=9 ; p>0.5$ for each parameter). On average, the amplitude of subthreshold PSPs and the firing probability on evoked responses changed by $<2 \%$ during control experiments (Fig. 5B2).

The functional impact of the $10 \mathrm{~Hz}$ conditioning on sensoryevoked responses was examined in nine of the cells experiencing LLPIE. The probability that whisker-evoked PSPs elicited an AP was augmented after the induction protocol in all cells (before, $0.25 \pm 0.09$ vs after, $0.45 \pm 0.09, n=9 ; p<0.01$ ) (Fig. $5 C, D$ ). On average, the firing probability was enhanced by $157.2 \pm 56.1 \%$ $(n=9)$ 15-20 min after induction. This facilitation in PSP-spike coupling, which persisted throughout the postconditioning period, was not associated with changes in the membrane potential measured at the foot of the evoked PSPs (before, $-70.6 \pm 1.5 \mathrm{mV}$ vs after, $-70.3 \pm 1.5 \mathrm{mV}, n=9 ; p>0.7)$. To determine whether the increase in spike firing on sensory responses was due to a reinforcement of the synaptic strength, we compared the amplitude of subthreshold PSPs in control and after conditioning (Fig. $5 E$ ). As shown by the overlaid of averaged responses in control and after induction, amplitude and rising slope of subthreshold PSPs were not affected by the conditioning $(n=9 ; p>0.5$ for both parameters) (Fig. $5 E$, right). The constancy in amplitude and shape of SEPs before and after conditioning is also consistent with the absence of changes in synaptic efficacy observed at the level of individual neurons (Fig. $5 E$ ). To elucidate the origin of the increase in spike probability, we compared the values of AP 

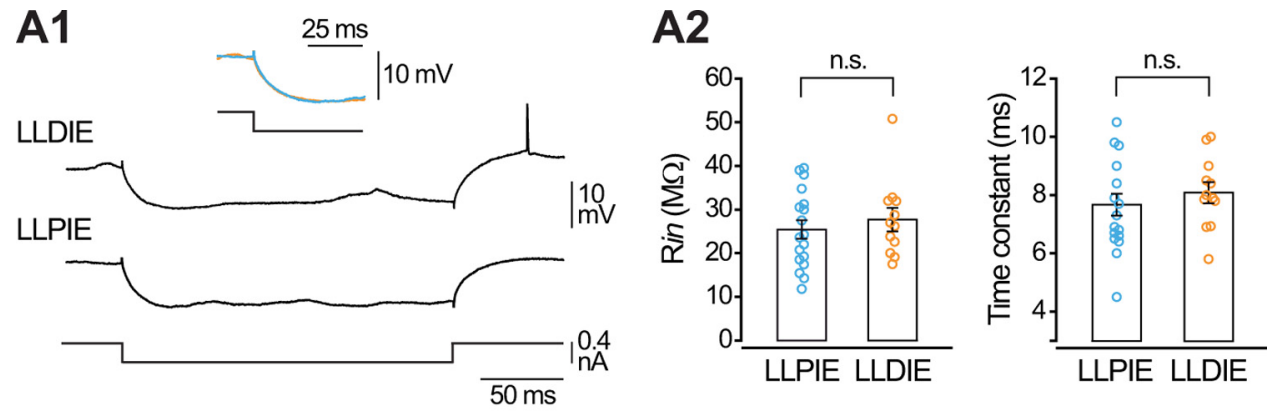

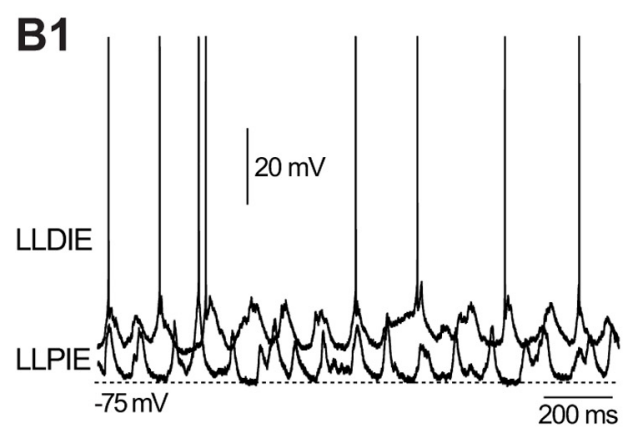

B2
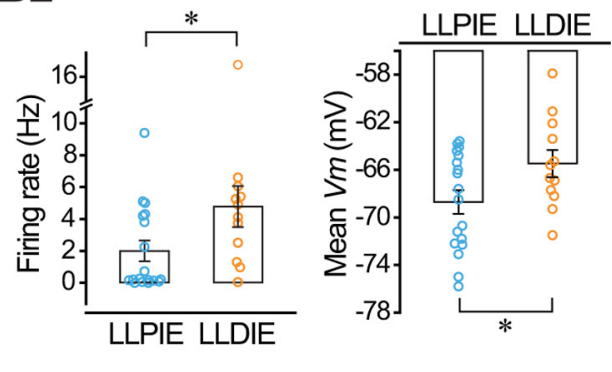

C1

LLPIE

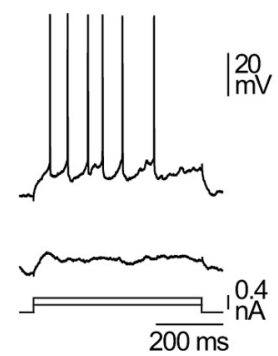

LLDIE
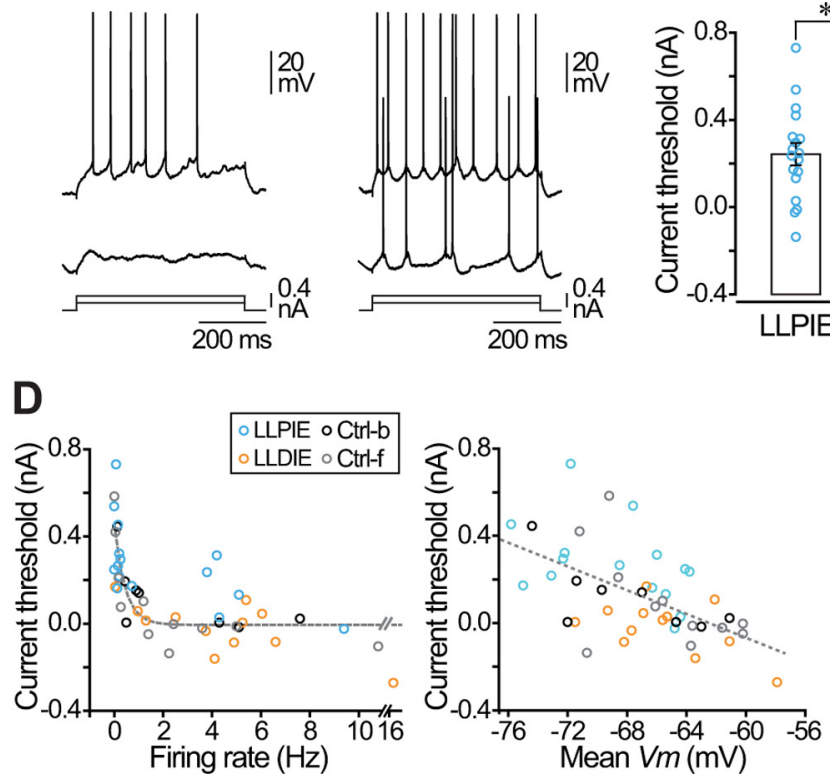

C2
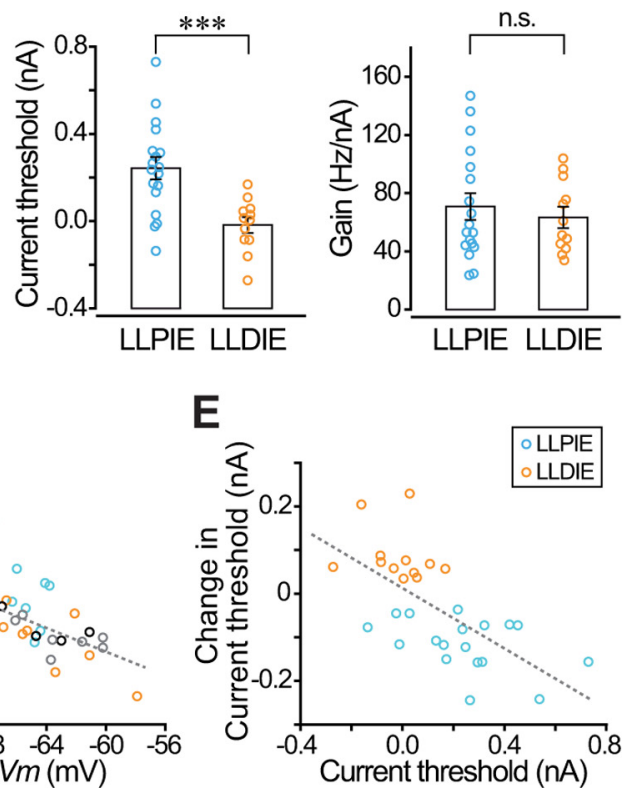

Figure 4. The sign of intrinsic plasticity correlates with baseline level of spontaneous discharge and excitability. $\boldsymbol{A}$, Potentiated and depressed neurons exhibit similar passive membrane properties. $\boldsymbol{A} 1$, Average voltage responses $(n \geq 10)$ to hyperpolarizing $(-0.4 \mathrm{nA})$ current pulses obtained during control periods in a potentiated (LLPIE) and a depressed (LLDIE) neuron. The inset is the superimposed expansion of the initial part of responses shown below. $A 2$, Pooled data of control $R_{\text {in }}$ and membrane time constant values from potentiated ( $\left.n=18\right)$ and depressed $(n=12)$ cells. $\boldsymbol{B}$, Pyramidal neurons expressing LLPIE are more hyperpolarized and less active than neurons expressing LLDIE. B1, Representative epochs of spontaneous activity (1.5 s recordings) recorded during control periods in barrel cortex pyramidal neurons exhibiting either LLPIE or LLDIE after induction. B2, Corresponding summary data (18 potentiated and 12 depressed neurons) of baseline spontaneous firing rate and mean $V_{\mathrm{m}}$. C, Current threshold is lower in neurons experiencing LLDIE. C1, Examples of control voltage responses in a potentiated and a depressed neuron to the injection of current pulses of same intensity. C2, Population data of control current threshold and gain in LLPIE $(n=18)$ and LLDIE $(n=12)$ neurons. D, Relationship between control current threshold values and baseline spontaneous firing rate (left) and mean membrane potential (right), for control cells recorded under barbiturate (Crtl-b, black symbols, $n=9$ ), control cells under fentanyl (Ctrl-f, gray symbols, $n=11$ ), and for neurons experiencing potentiation (LLPIE, blue symbols, $n=18$ ) or depression (LLDIE, orange symbols, $n=12$ ) of their excitability. Dashed gray lines are best curve fits. $E$, Population data (18 potentiated and 12 depressed neurons) showing that the bidirectional changes in threshold current after induction correlated well $(r=-0.65)$ with the initial threshold current value. ${ }^{*} p<0.05 ;{ }^{* * *} p<0.001 ;$ n.s., nonsignificant.

threshold voltage on individual suprathreshold responses before and after LLPIE. This analysis was performed in cells with sufficient number of suprathreshold PSPs during baseline periods to allow a reliable comparison with conditioned responses $(n=8)$. The conditioning procedure caused a significant reduction in spike threshold in all cases (before, $-53.7 \pm 0.9 \mathrm{mV}$ vs after,
$-55.0 \pm 0.7 \mathrm{mV}, n=8 ; p<0.001$ ) (Fig. $5 F$ ). The mean spike latency, measured from the onset of the air-puff to the peak of AP, was also significantly reduced by the conditioning (before, $20.3 \pm$ $0.9 \mathrm{~ms}$ vs after, $19.6 \pm 0.8 \mathrm{~ms}, n=8$; $p<0.05$ ) (Fig. $5 F$ ). Consistent with results from current-evoked responses, the lowering of spike threshold was correlated with an increase in the slope of 
A

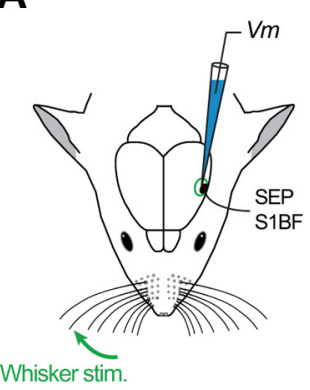

B1

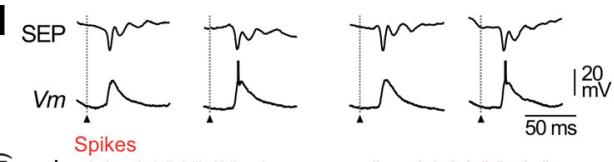

B2

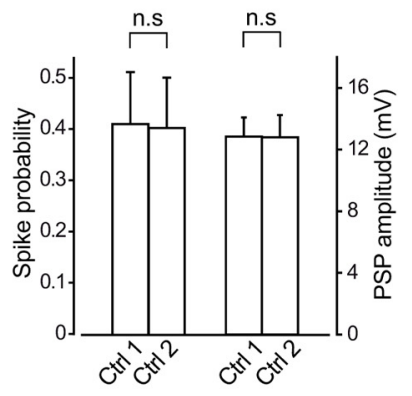

C

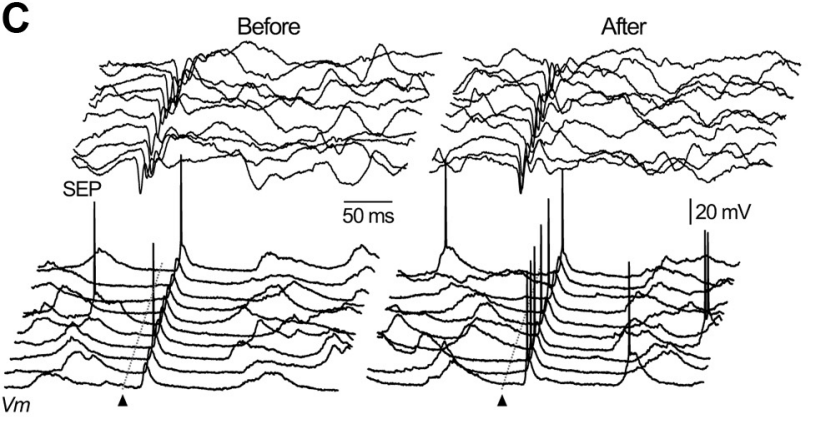

E
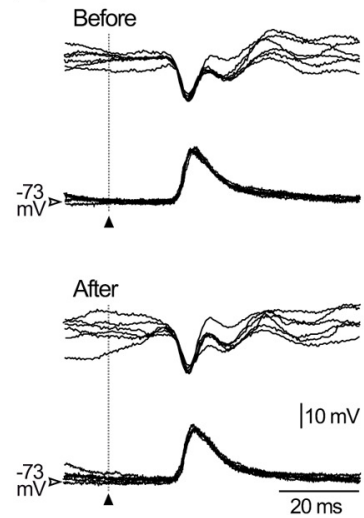
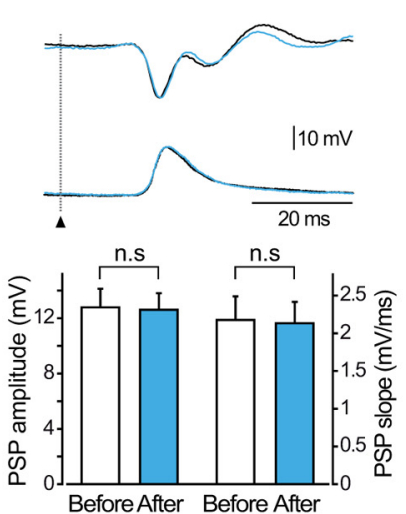

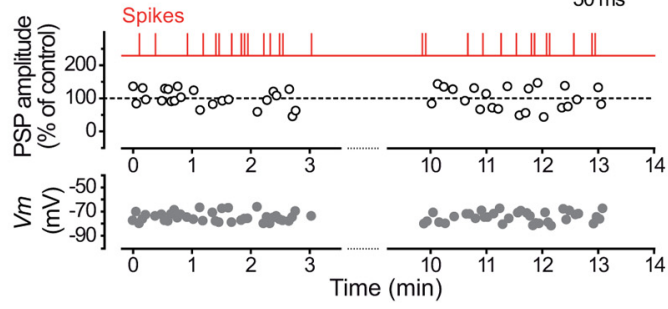

D

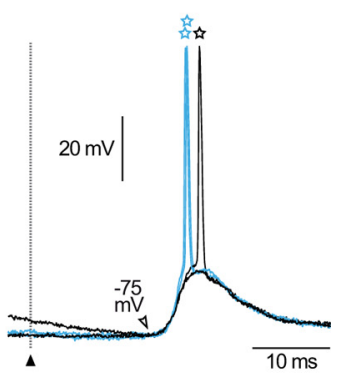

$\mathbf{F}$
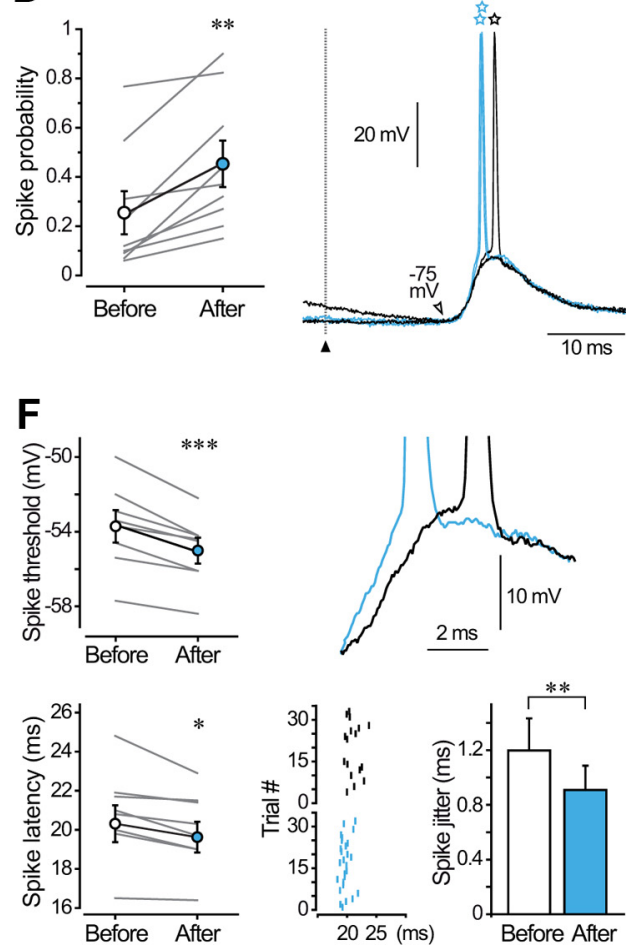

Figure 5. The responsiveness of barrel cortex neurons to sensory inputs is enhanced during LLPIE. $A$, SEPs at the surface of the barrel cortex and related postsynaptic depolarizations in layer 5 pyramidal neurons $\left(V_{m}\right)$ were recorded in response to air-puff stimuli applied to the contralateral whiskers (Whisker stim.). B, Stability of whisker-evoked sensory responses in unconditioned neurons. B1, Time course of membrane potential (measured $3 \mathrm{~ms}$ before the onset of the evoked potentials, $V_{\mathrm{m}^{\prime}}$ gray symbols) and amplitude of evoked subthreshold PSPs (white symbols) during two consecutive control periods of stimulations. PSP amplitude is normalized to the mean value of the first control period. Red vertical lines indicate the occurrence of suprathreshold evoked responses. The onset of whisker stimulations is indicated by filled triangles here and in subsequent figures. The top traces show typical examples of subthreshold and suprathreshold intracellular responses together with related SEPS. B2, Summary plot showing that firing probability on evoked potentials and amplitude of subthreshold PSPs remained stable ( $p>0.5$ for both parameters) during control periods ( $n=9$ neurons). C, Ten successive whisker-evoked (20 psi) intracellular responses and corresponding SEPs obtained before and after LLPIE induction. Voltage calibrations applied to both series of traces. $\boldsymbol{D}$, Population analysis ( $n=9$ neurons) comparing spike probability on sensory responses before and after the conditioning spike-trains. Right panel illustrates typical examples of whisker-evoked responses obtained in control (black traces) and after LLPIE (blue traces) clearly showing the enhanced firing probability and the decreased firing latency after induction (open stars). $\boldsymbol{E}$, Superimposition $(n=6)$ of subthreshold PSPs and corresponding SEPs recorded in control and postinduction periods. Voltage calibrations applied to both series of traces. The corresponding average responses are superimposed at right. Pooled data ( $n=9$ neurons, bottom graph) indicate that amplitude and rising slope of subthreshold PSPs were not significantly modified ( $p>0.5$ for both parameters) by the intracellular conditioning. $\boldsymbol{F}$, Population analysis ( $n=8$ neurons) showing that LLPIE was associated with a significant decrease in voltage threshold and latency of spikes on sensory-evoked PSPs (left part), as illustrated by the enlarged view of superimposed single responses from control (black trace) and postinduction periods (blue trace) shown at right. Bottom right, Raster plot obtained from a representative cortical neuron showing the time of occurrence of action potentials evoked by sensory stimuli before (black vertical lines, $n=17$ trials) and during LLPIE (blue vertical lines, $n=27$ trials). The bar graph shows that the temporal variability of spike latency is reduced after conditioning $\left(n=8\right.$ neurons). ${ }^{*} p<0.05$; ${ }^{* *} p<0.01$; ${ }^{* * *} p<0.001 ;$ i.s., nonsignificant. $\boldsymbol{C}-\boldsymbol{F}$ are from the same neuron.

the synaptic depolarization preceding the discharge (before, $3.45 \pm 0.55 \mathrm{mV} / \mathrm{ms}$ vs after, $3.71 \pm 0.59 \mathrm{mV} / \mathrm{ms}, n=8 ; p<0.05$ ), and the jitter of evoked spikes was reduced after induction $(n=8$; $p<0.01$ ), indicating an enhanced temporal precision of AP discharge on sensory responses (Fig. $5 F$ ).

Opposite results were obtained in cortical neurons expressing depression of their intrinsic excitability after conditioning. In these cells, the probability that a sensory-evoked PSP initiated an AP was $0.47 \pm 0.14$ before and $0.23 \pm 0.09$ after induction $(n=6$; $p<0.01$ ), corresponding to a reduction in firing probability of $59.2 \pm 8.1 \%(n=6)$ (Fig. 6A, $B)$. Again, the membrane potential value just before the onset of the evoked responses remained stable $(n=6 ; p>0.3)$ after conditioning, indicating that the decrease in spike probability was not due to a membrane hyperpolarization. As observed for potentiated responses, and despite a trial-to-trial variability, amplitude (101.1 $\pm 1.9 \%$ of the control, $n=6 ; p>0.6)$ and rising slope $(101.5 \pm 2.9 \%, n=6 ; p>0.7)$ of subthreshold synaptic potentials were not significantly modified 
A
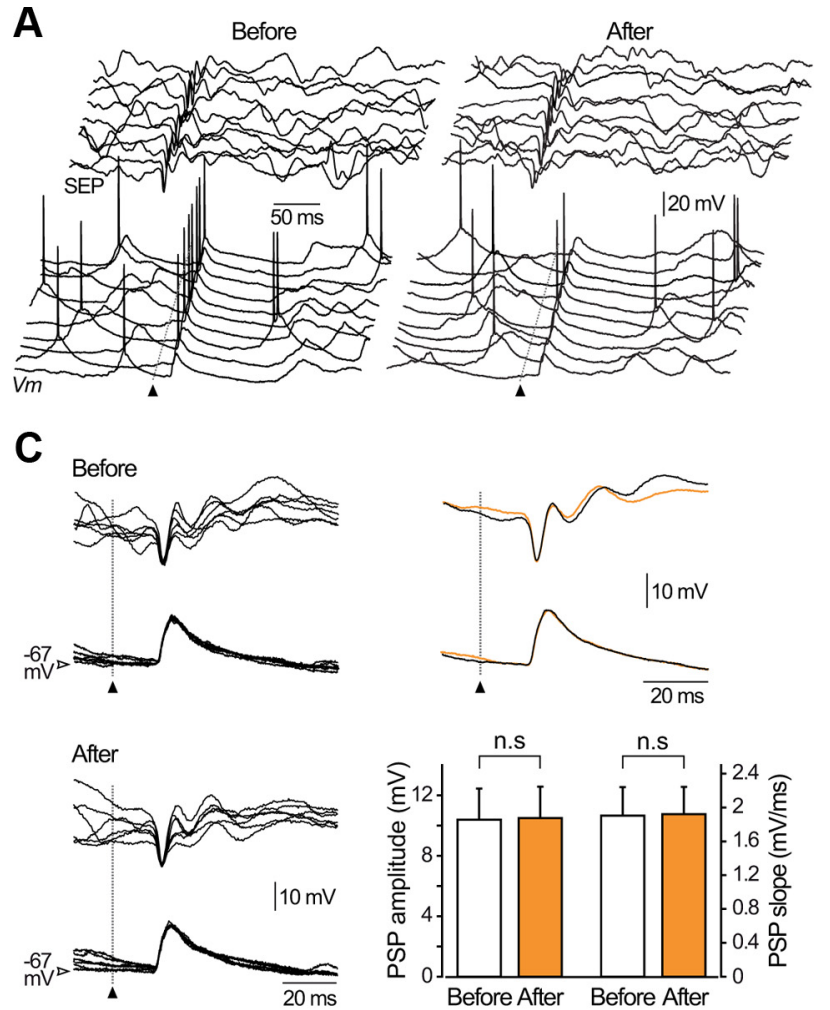
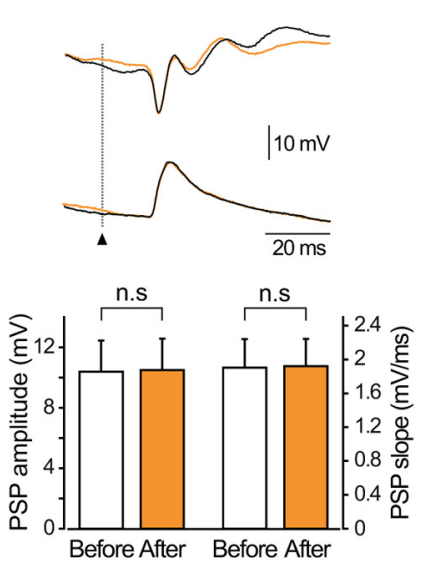

B
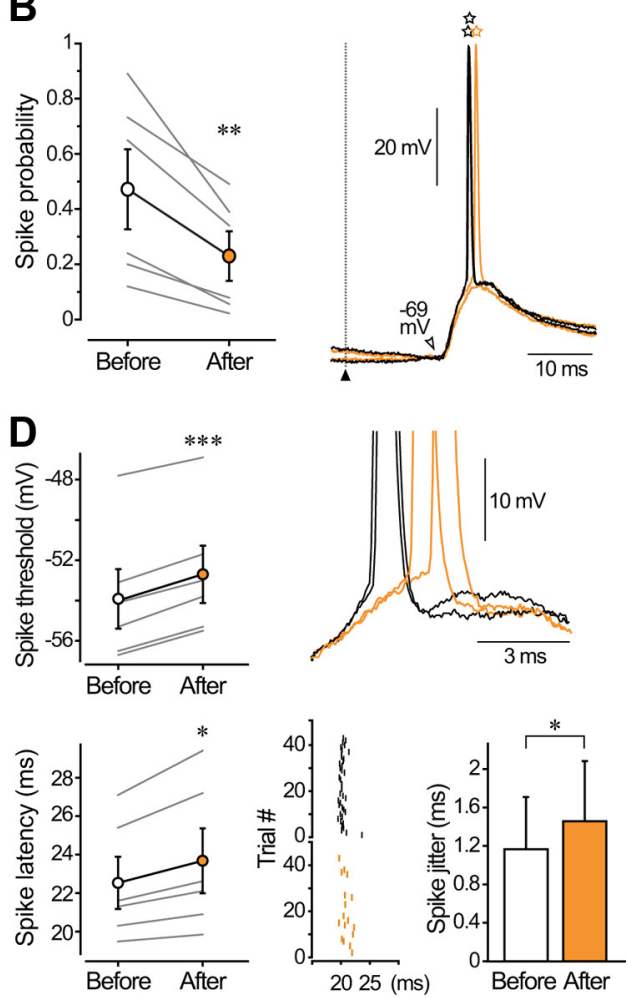

Figure 6. The responsiveness to sensory inputs is reduced in barrel cortex neurons experiencing LLDIE. $A$, Ten successive whisker-evoked (20 psi) intracellular responses and corresponding SEPs obtained before and after LLDIE induction. Voltage calibrations applied to both series of traces. $\boldsymbol{B}$, Population analysis ( $n=6$ depressed neurons) comparing spike probability on sensory responses before and after the conditioning spike-trains. Right, Typical examples of whisker-related PSPs in control (black traces) and during LLDIE (orange traces) showing the increased firing latency (open stars) on sensory-evoked responses after induction. C, Superimposition $(n=6)$ of subthreshold PSPs and corresponding SEPs recorded in control and postinduction periods. Voltage calibrations applied to both series of traces. Corresponding average responses are superimposed at right. Pooled data ( $n=6$ neurons, bottom graph) showing that amplitude and rising slope of subthreshold PSPs were not significantly modified ( $p>0.6$ for both parameters) by the intracellular conditioning. $\boldsymbol{D}$, Population analysis (left part, $n=6$ neurons) showing that LLDIE was associated with an augmentation of voltage threshold and latency of spikes on evoked PSPs, as illustrated at right by the enlarged view of superimposed responses obtained in control (black traces) and postinduction periods (orange traces). Bottom right, Raster plot showing the time of occurrence of sensory-evoked action potentials before (black vertical lines, $n=40$ trials) and after (orange vertical lines, $n=$ 17 trials) LLDIE induction in a barrel cortex pyramidal neuron. The bar graph indicates that the temporal variability of spike latency is enhanced after conditioning $\left(n=6\right.$ neurons). ${ }^{*} p<0.05 ;{ }^{* *} p<$ $0.01 ; * * * 0.001 ;$ n.S., nonsignificant. $\boldsymbol{A}-\boldsymbol{D}$ are from the same neuron.

by the conditioning (Fig. 6C). This demonstrates that decreased responsiveness to sensory stimuli does not result from a change in the efficacy of synaptic inputs onto the recorded cells. As it decreased the efficiency of sensory stimulations, intracellular conditioning significantly decreased the voltage spike threshold (before, $-53.9 \pm 1.5 \mathrm{mV}$ vs after, $-52.7 \pm 1.4 \mathrm{mV}, n=6$; $p<$ 0.001 ) and slowed the synaptic depolarization preceding the discharge (before, $3.35 \pm 0.65 \mathrm{mV} / \mathrm{ms}$ vs after, $3.02 \pm 0.72 \mathrm{mV} / \mathrm{ms}$, $n=6 ; p<0.01$ ) (Fig. 6D). In contrast to potentiated cells, latency and temporal variability of AP discharge on evoked synaptic potentials were enhanced after induction of LLDIE $(n=6 ; p<0.05)$ (Fig. 6D).

We conclude that induction of LLPIE or LLDIE is associated with opposite changes in the reliability and precision of sensoryinduced firing in pyramidal neurons, suggesting that intrinsic plasticity may contribute to the control of sensory information processing in the adult barrel cortex.

\section{Discussion}

We showed that a few minutes of $10 \mathrm{~Hz}$ firing can trigger a rapid and persistent increase or decrease in the overall excitability of layer 5 pyramidal barrel cortex neurons in vivo. LLPIE was expressed as reduction in threshold current leading to a leftward shift in the entire F-I relationship. Conversely, LLDIE consisted of an augmented firing threshold and a rightward shift of the input-output function. This activity-dependent bidirectional modification of intrinsic excitability was not associated with changes in passive membrane properties. Induction of LLPIE and LLDIE resulted, respectively, in an increased or a decreased cell firing in response to whisker-evoked inputs, without changes in the underlying synaptic strength. These data suggest that bidirectional intrinsic plasticity is involved in the use-dependent adaptation of sensory responses in barrel cortex.

\section{Bidirectional intrinsic plasticity in barrel cortex neurons in vivo}

Bidirectional intrinsic plasticity has already been described from in vitro and in vivo preparations. In hippocampal slices, the induction of long-term synaptic potentiation or depression by specific conditioning protocols can be associated with bidirectional changes in intrinsic excitability. These cellular modifications were found to act in synergy with synaptic changes (Daoudal et al., 2002; Li et al., 2004) or took the form of negative feedback mechanisms to normalize output firing (Fan et al., 2005; Brager and Johnston, 2007). Remarkably, we found that the same conditioning protocol could induce either LLPIE or LLDIE in barrel cortex neurons in vivo. We recently made partly similar observations in motor cortex neurons in vivo where repeated injection of suprathreshold currents produced enduring increases or decreases in membrane excitability, which 
were associated with changes in both current threshold and neuronal gain (Paz et al., 2009). Here, we show that the variability in neuronal spontaneous firing rate, which is inherent to the living brain (Sachdev et al., 2004; Polack and Charpier, 2006; Jacob et al., 2007; Pidoux et al., 2011), could provide a significant determinant of the polarity of plasticity. Indeed, we established that depressed cortical neurons exhibited, during control periods, a higher spontaneous firing rate and a more depolarized $V_{\mathrm{m}}$ compared with potentiated cells. We also provided evidence that depressed cells were characterized by a lower average value of control threshold current. This suggests that a major contributor to the relatively elevated spontaneous firing of depressed neurons is an enhanced intrinsic excitability at the time of control recording. Phosphorylation of voltage-gated ionic channels by protein kinases is known to regulate neuronal excitability in various cell types (Cantrell and Catterall, 2001). The cell-to-cell variability in baseline excitability and spontaneous firing may thus reflect heterogeneity in the activity of ionic channels. Notably, the excitability of silent and spontaneously active vestibular nucleus neurons was found to be differentially affected by the dialysis of constitutively activated calcium/calmodulin-dependent protein kinase II, suggesting differences in the phosphorylation state of ionic channels in relation with the level of activity (Nelson et al., 2005). Our results, which clearly show an inverse relationship between the sign of intrinsic plasticity and the control level of cellular excitability and firing, suggest that bidirectional intrinsic plasticity could represent a dynamic mechanism for context-dependent homeostatic-like regulation of excitability in barrel cortex. However, future experiments are required to determine whether bidirectional plasticity can be induced in a single cortical neuron by artificially manipulating the level of spontaneous discharge.

\section{Cellular mechanisms underlying bidirectional intrinsic plasticity}

The specific changes in threshold current associated with LLPIE or LLDIE suggest that intrinsic plasticity is mediated by modifications in voltage-dependent ionic conductances available around spike threshold. This is also supported by the lack of changes in somatic $R_{\text {in }}$ and by the stability in the amplitude and kinetics of subthreshold PSPs after conditioning. We also showed that modifications in current-induced firing were accompanied by alterations in voltage threshold of the first AP. As previously reported (Azouz and Gray, 2000), the voltage threshold was negatively correlated with the slope of membrane depolarization preceding the AP. Biophysical models indicate that this inverse relationship is conferred by the kinetic properties of $\mathrm{Na}^{+}$and $\mathrm{K}^{+}$ conductances (Noble and Stein, 1966). Thus, although the identity of affected ionic conductances remains to be determined, changes in $\mathrm{Na}^{+}$and/or $\mathrm{K}^{+}$channel properties provide plausible mechanisms for LLPIE and LLDIE. Accordingly, a decrease in threshold current accompanying potentiation of membrane excitability in optic tectal neurons of the Xenopus tadpole (Aizenman et al., 2003) and in hippocampal neurons (Ganguly et al., 2000; Li et al., 2004; Xu et al., 2005) has been attributed to changes in $\mathrm{Na}^{+}$ currents. Upregulation or downregulation of slowly inactivating $\mathrm{K}^{+}$currents have also been involved in the modulation of AP threshold and global excitability in hippocampal cells in cultures (Li et al., 2004) and in striatal neurons in vivo (Mahon et al., 2003). Finally, a decrease in threshold current in cultured cortical neurons after chronic deprivation of synaptic inputs results from concomitant changes in voltage-gated $\mathrm{Na}^{+}$and $\mathrm{K}^{+}$conductances (Desai et al., 1999).

Previous work indicates that induction and maintenance of intrinsic plasticity usually requires an increase in intracellular calcium concentration and subsequent activation of $\mathrm{Ca}^{2+}$ dependent signaling cascades (Xu and Kang, 2005; Delord et al., 2007). In the present study, the repeated firing during conditioning likely triggered calcium influx. The direction of plasticity could depend on the activated signaling pathway since neurons with higher spontaneous firing may have an elevated resting level of calcium setting a different balance of downstream kinases and phosphatases (Nelson et al., 2005). Supporting this hypothesis, a bidirectional control of the open probability of large conductance $\mathrm{K}^{+}$channels by different proteins kinases has been found in vestibular nucleus neurons (van Welie and du Lac, 2011). Moreover, induction of potentiation and depression of membrane excitability in hippocampal neurons both require calcium influx, but engage divergent sources of calcium increase, enzymatic cascades, or ionic conductances (Ganguly et al., 2000; Li et al., 2004).

\section{Long-lasting modulation of sensory inputs}

Importantly, we report that potentiation and depression of barrel cortex neuron excitability were accompanied, respectively, by an increase or a decrease in spike discharge probability on whiskerevoked synaptic responses. This bidirectional modulation of firing reliability is reminiscent of the bidirectional regulation of EPSP-spike coupling observed in hippocampal neurons during synaptic plasticity. Long-term potentiation of excitatory transmission in CA1 hippocampal neurons is accompanied by an enhanced probability of discharge in response to a given synaptic potential (Bliss and Lomo, 1973), a phenomenon partly due to an increase in intrinsic excitability (Chavez-Noriega et al., 1990; Daoudal et al., 2002). Conversely, a decrease in EPSP-spike coupling can be concomitantly induced with synaptic depression (Daoudal et al., 2002). Contrasting with these parallel changes in synaptic and intrinsic properties, we found here that amplitude and kinetics of subthreshold whiskerevoked PSPs remained unchanged after conditioning. This suggests that the modulation of sensory input efficiency resulted solely from modifications in intrinsic currents that govern neuronal discharge. Changes in firing properties on synaptic responses could have been directly predicted from the changes in excitability. Indeed, the leftward shift in $F-I$ relationship during LLPIE is expected to increase neuronal sensitivity to depolarizing inputs, making previously subthreshold postsynaptic events efficient for spike initiation. Conversely, the rightward shift in F-I relationship during LLDIE will tend to decrease the ease with which a spike can be elicited by a given synaptic input. Moreover, facilitation of firing on both current- and whisker-evoked responses was associated with a reduction in voltage spike threshold, a decrease in AP latency, and an increase in the temporal precision of spiking, while reduction in firing probability led to opposite changes in AP properties. Thus, our findings strongly suggest that activity-dependent plasticity of intrinsic excitability results in a bidirectional control of sensory information processing in the barrel cortex.

\section{Functional implications of bidirectional intrinsic plasticity}

Synaptic plasticity has been proposed to underlie experiencedependent refinement of cortical responsiveness (Feldman, 2009), but the functional significance of intrinsic plasticity remains unclear. Changes in barrel cortex neuron intrinsic excit- 
ability have been mainly viewed as compensatory mechanisms to counteract loss of synaptic inputs during sensory deprivation (Breton and Stuart, 2009; Sun, 2009).

Here, we report that layer 5 barrel cortex neurons excitability can be modulated in a bidirectional manner by episodes of patterned firing resembling their activity during whisking. By lowering the minimal synaptic input required to generate cell firing, LLPIE could contribute to the strengthening of cortical responsiveness observed following repeated coactivation of specific whiskers or behavioral training (Diamond et al., 1994; Wiest et al., 2010). In addition, as shown in hippocampus (Cudmore et al., 2010), the enhanced spike-time precision after LLPIE induction could increase the propensity for synchronization in the cortical network. This persistent increase in firing reliability within the conditioned network might also promote the spread of excitation leading to functional reconfiguration of sensory receptive fields. Symmetrical changes are expected to occur during LLDIE. Thus, during whisker-related sensorimotor learning, induction of LLPIE or LLDIE in distinct corticofugal layer 5 neurons could act in synergy with synaptic plasticity to set and fix adapted patterns of activity within the barrel cortex, allowing the appropriate activation of downstream sensory or motors systems.

\section{References}

Aizenman CD, Akerman CJ, Jensen KR, Cline HT (2003) Visually driven regulation of intrinsic neuronal excitability improves stimulus detection in vivo. Neuron 39:831-842.

Azouz R, Gray CM (2000) Dynamic spike threshold reveals a mechanism for synaptic coincidence detection in cortical neurons in vivo. Proc Natl Acad Sci U S A 97:8110-8115.

Bekisz M, Garkun Y, Wabno J, Hess G, Wrobel A, Kossut M (2010) Increased excitability of cortical neurons induced by associative learning: an ex vivo study. Eur J Neurosci 32:1715-1725.

Bliss TV, Lomo T (1973) Long-lasting potentiation of synaptic transmission in the dentate area of the anaesthetized rabbit following stimulation of the perforant path. J Physiol 232:331-356.

Brager DH, Johnston D (2007) Plasticity of intrinsic excitability during long-term depression is mediated through mGluR-dependent changes in $\mathrm{I}(\mathrm{h})$ in hippocampal CA1 pyramidal neurons. J Neurosci 27:13926-13937.

Breton JD, Stuart GJ (2009) Loss of sensory input increases the intrinsic excitability of layer 5 pyramidal neurons in rat barrel cortex. J Physiol 587:5107-5119.

Cantrell AR, Catterall WA (2001) Neuromodulation of $\mathrm{Na}+$ channels: an unexpected form of cellular plasticity. Nat Rev Neurosci 2:397-407.

Carvell GE, Simons DJ (1990) Biometric analyses of vibrissal tactile discrimination in the rat. J Neurosci 10:2638-2648.

Chavez-Noriega LE, Halliwell JV, Bliss TV (1990) A decrease in firing threshold observed after induction of the EPSP-spike (E-S) component of long-term potentiation in rat hippocampal slices. Exp Brain Res 79:633-641.

Constantinople CM, Bruno RM (2011) Effects and mechanisms of wakefulness on local cortical networks. Neuron 69:1061-1068.

Crochet S, Petersen CC (2006) Correlating whisker behavior with membrane potential in barrel cortex of awake mice. Nat Neurosci 9:608-610.

Cudmore RH, Turrigiano GG (2004) Long-term potentiation of intrinsic excitability in LV visual cortical neurons. J Neurophysiol 92:341-348.

Cudmore RH, Fronzaroli-Molinieres L, Giraud P, Debanne D (2010) Spiketime precision and network synchrony are controlled by the homeostatic regulation of the D-type potassium current. J Neurosci 30:12885-12895.

Daoudal G, Debanne D (2003) Long-term plasticity of intrinsic excitability: learning rules and mechanisms. Learn Mem 10:456-465.

Daoudal G, Hanada Y, Debanne D (2002) Bidirectional plasticity of excitatory postsynaptic potential (EPSP)-spike coupling in CA1 hippocampal pyramidal neurons. Proc Natl Acad Sci U S A 99:14512-14517.

Delord B, Berry H, Guigon E, Genet S (2007) A new principle for information storage in an enzymatic pathway model. PLoS Comput Biol 3:e124.

Desai NS, Rutherford LC, Turrigiano GG (1999) Plasticity in the intrinsic excitability of cortical pyramidal neurons. Nat Neurosci 2:515-520.
Diamond ME, Armstrong-James M, Ebner FF (1993) Experiencedependent plasticity in adult rat barrel cortex. Proc Natl Acad Sci U S A 90:2082-2086.

Diamond ME, Huang W, Ebner FF (1994) Laminar comparison of somatosensory cortical plasticity. Science 265:1885-1888.

Fan Y, Fricker D, Brager DH, Chen X, Lu HC, Chitwood RA, Johnston D (2005) Activity-dependent decrease of excitability in rat hippocampal neurons through increases in I(h). Nat Neurosci 8:1542-1551.

Fee MS, Mitra PP, Kleinfeld D (1997) Central versus peripheral determinants of patterned spike activity in rat vibrissa cortex during whisking. J Neurophysiol 78:1144-1149.

Feldman DE (2009) Synaptic mechanisms for plasticity in neocortex. Annu Rev Neurosci 32:33-55.

Fox K (2002) Anatomical pathways and molecular mechanisms for plasticity in the barrel cortex. Neuroscience 111:799-814.

Fox K (2008) Barrel cortex. Cambridge, UK: Cambridge UP.

Fricker D, Verheugen JA, Miles R (1999) Cell-attached measurements of the firing threshold of rat hippocampal neurones. J Physiol 517:791-804.

Ganguly K, Kleinfeld D (2004) Goal-directed whisking increases phaselocking between vibrissa movement and electrical activity in primary sensory cortex in rat. Proc Natl Acad Sci U S A 101:12348-12353.

Ganguly K, Kiss L, Poo M (2000) Enhancement of presynaptic neuronal excitability by correlated presynaptic and postsynaptic spiking. Nat Neurosci 3:1018-1026.

Jacob V, Brasier DJ, Erchova I, Feldman D, Shulz DE (2007) Spike timingdependent synaptic depression in the in vivo barrel cortex of the rat. J Neurosci 27:1271-1284.

Kleinfeld D, Ahissar E, Diamond ME (2006) Active sensation: insights from the rodent vibrissa sensorimotor system. Curr Opin Neurobiol $16: 435-444$.

Li CY, Lu JT, Wu CP, Duan SM, Poo MM (2004) Bidirectional modification of presynaptic neuronal excitability accompanying spike timingdependent synaptic plasticity. Neuron 41:257-268.

Mahon S, Deniau JM, Charpier S (2001) Relationship between EEG potentials and intracellular activity of striatal and cortico-striatal neurons: an in vivo study under different anesthetics. Cereb Cortex 11:360-373.

Mahon S, Casassus G, Mulle C, Charpier S (2003) Spike-dependent intrinsic plasticity increases firing probability in rat striatal neurons in vivo. J Physiol 550:947-959.

Maravall M, Stern EA, Svoboda K (2004) Development of intrinsic properties and excitability of layer $2 / 3$ pyramidal neurons during a critical period for sensory maps in rat barrel cortex. J Neurophysiol 92:144-156.

Marder E, Prinz AA (2002) Modeling stability in neuron and network function: the role of activity in homeostasis. Bioessays 24:1145-1154.

Matthews EA, Weible AP, Shah S, Disterhoft JF (2008) The BK-mediated fAHP is modulated by learning a hippocampus-dependent task. Proc Natl Acad Sci U S A 105:15154-15159.

Nelson AB, Gittis AH, du Lac S (2005) Decreases in CaMKII activity trigger persistent potentiation of intrinsic excitability in spontaneously firing vestibular nucleus neurons. Neuron 46:623-631.

Noble D, Stein RB (1966) The threshold conditions for initiation of action potentials by excitable cells. J Physiol 187:129-162.

Paxinos G, Watson C (1986) The rat brain in stereotaxic coordinates. Sydney: Academic.

Paz JT, Mahon S, Tiret P, Genet S, Delord B, Charpier S (2009) Multiple forms of activity-dependent intrinsic plasticity in layer $\mathrm{V}$ cortical neurones in vivo. J Physiol 587:3189-3205.

Petersen CC (2007) The functional organization of the barrel cortex. Neuron 56:339-355.

Petersen CC, Hahn TT, Mehta M, Grinvald A, Sakmann B (2003) Interaction of sensory responses with spontaneous depolarization in layer $2 / 3$ barrel cortex. Proc Natl Acad Sci U S A 100:13638-13643.

Pidoux M, Mahon S, Deniau JM, Charpier S (2011) Integration and propagation of somatosensory responses in the corticostriatal pathway: an intracellular study in vivo. J Physiol 589:263-281.

Polack PO, Charpier S (2006) Intracellular activity of cortical and thalamic neurones during high-voltage rhythmic spike discharge in Long-Evans rats in vivo. J Physiol 571:461-476.

Saar D, Grossman Y, Barkai E (1998) Reduced after-hyperpolarization in rat piriform cortex pyramidal neurons is associated with increased learning capability during operant conditioning. Eur J Neurosci 10:1518-1523. 
Sachdev RN, Ebner FF, Wilson CJ (2004) Effect of subthreshold up and down states on the whisker-evoked response in somatosensory cortex. J Neurophysiol 92:3511-3521.

Schubert D, Staiger JF, Cho N, Kötter R, Zilles K, Luhmann HJ (2001) Layer-specific intracolumnar and transcolumnar functional connectivity of layer V pyramidal cells in rat barrel cortex. J Neurosci 21:3580-3592.

Schubert D, Kötter R, Luhmann HJ, Staiger JF (2006) Morphology, electrophysiology and functional input connectivity of pyramidal neurons characterizes a genuine layer va in the primary somatosensory cortex. Cereb Cortex 16:223-236.

Siucinska E, Kossut M (1996) Short-lasting classical conditioning induces reversible changes of representational maps of vibrissae in mouse SI cortex-a 2DG study. Cereb Cortex 6:506-513.

Siucinska E, Kossut M (2004) Experience-dependent changes in cortical whisker representation in the adult mouse: a 2-deoxyglucose study. Neuroscience 127:961-971.

Skibinska-Kijek A, Radwanska A, Kossut M (2008) Alpha calcium/calmodulin dependent protein kinase II in learning-dependent plasticity of mouse somatosensory cortex. Neuroscience 151:750-757.

Sourdet V, Russier M, Daoudal G, Ankri N, Debanne D (2003) Long-term enhancement of neuronal excitability and temporal fidelity mediated by metabotropic glutamate receptor subtype 5. J Neurosci 23:10238-10248.

Sun QQ (2009) Experience-dependent intrinsic plasticity in interneurons of barrel cortex layer IV. J Neurophysiol 102:2955-2973.

van Welie I, du Lac S (2011) Bidirectional control of BK channel open probability by CaMKIIand PKC in medial vestibular nucleus neurons. J Neurophysiol 105:1651-1659.

Wiest MC, Thomson E, Pantoja J, Nicolelis MA (2010) Changes in S1 neural responses during tactile discrimination learning. J Neurophysiol 104: $300-312$.

Wilent WB, Contreras D (2004) Synaptic responses to whisker deflections in rat barrel cortex as a function of cortical layer and stimulus intensity. J Neurosci 24:3985-3998.

Xu J, Kang J (2005) The mechanisms and functions of activity-dependent long-term potentiation of intrinsic excitability. Rev Neurosci 16:311-323.

Xu J, Kang N, Jiang L, Nedergaard M, Kang J (2005) Activity-dependent long-term potentiation of intrinsic excitability in hippocampal CA1 pyramidal neurons. J Neurosci 25:1750-1760.

Zhu JJ, Connors BW (1999) Intrinsic firing patterns and whisker-evoked synaptic responses of neurons in the rat barrel cortex. J Neurophysiol 81:1171-1183. 